\title{
Total synthesis of the potent antitumor polyketide (-)-callystatin $A^{\#}$
}

\author{
Luiz C. Dias,* and Paulo R. R. Meira \\ Instituto de Química, Universidade Estadual de Campinas, UNICAMP \\ C.P. 6154, 13084-971, Campinas, SP, Brazil \\ E-mail: ldias@iqm.unicamp.br
}

\# Dedicated to Prof. Peter Bakuzis for his outstanding contributions to the field of synthetic organic chemistry in Brazil

\section{Supporting Information}

\begin{tabular}{|c|c|}
\hline CONTENTS & PAGE \\
\hline${ }^{1} \mathrm{H}$ NMR (300 MHz, $\left.\mathrm{CDCl}_{3}\right)$ - Ester (14) & SI-44 \\
\hline${ }^{13} \mathrm{C}$ NMR (75 MHz, CDCl 3 ) - Ester (14) & SI-44 \\
\hline DEPT 90/135 (75 MHz, CDCl 3 ) - Ester (14) & SI-45 \\
\hline IR (film) - Ester (14) & SI-45 \\
\hline${ }^{1} \mathrm{H}$ NMR (300 MHz, $\mathrm{CDCl}_{3}$ ) - Ester (15) & SI-46 \\
\hline${ }^{13} \mathrm{C}$ NMR (75 MHz, CDCl 3 ) - Ester (15) & SI-47 \\
\hline DEPT 90/135 (75 MHz, CDCl 3 ) - Ester (15) & SI-47 \\
\hline IR (film) - Ester (15) & SI-48 \\
\hline${ }^{1} \mathrm{H}$ NMR (300 MHz, $\left.\mathrm{CDCl}_{3}\right)$ - Lactone (13) & SI-49 \\
\hline${ }^{1} \mathrm{H}$ NMR (300 MHz, $\left.\mathrm{CDCl}_{3}\right)$ - TBS-Lactone from (13) & SI-49 \\
\hline${ }^{13} \mathrm{C} \mathrm{NMR} \mathrm{(75} \mathrm{MHz,} \mathrm{CDCl} 3$ ) - TBS-Lactone from (13) & SI-50 \\
\hline DEPT 90/135 (75 MHz, $\left.\mathrm{CDCl}_{3}\right)$ - TBS-Lactone from (13) & SI-50 \\
\hline IR (Film) - TBS-Lactone from (13) & SI-51 \\
\hline${ }^{1} \mathrm{H}$ NMR (300 MHz, $\left.\mathrm{CDCl}_{3}\right)$ - iPr-Lactol (17) & SI-51 \\
\hline${ }^{13} \mathrm{C}$ NMR (75 MHz, $\mathrm{CDCl}_{3}$ ) - iPr-Lactol (17) & SI-52 \\
\hline DEPT 90/135 (75 MHz, CDCl $\left.{ }_{3}\right)$ - iPr-Lactol (17) & SI-52 \\
\hline
\end{tabular}




\begin{tabular}{|c|c|}
\hline IR (Film) - iPr-Lactol (17) & SI-53 \\
\hline${ }^{1} \mathrm{H}$ NMR (300 MHz, $\left.\mathrm{CDCl}_{3}\right)$ - Alcohol from (17) & SI-53 \\
\hline${ }^{13} \mathrm{C}$ NMR (75 MHz, $\left.\mathrm{CDCl}_{3}\right)$ - Alcohol from (17) & SI-54 \\
\hline${ }^{1} \mathrm{H}$ NMR (300 MHz, $\left.\mathrm{CDCl}_{3}\right)$ - Unsaturated ester (20) & SI-55 \\
\hline${ }^{13} \mathrm{C}$ NMR (75 MHz, $\mathrm{CDCl}_{3}$ ) - Unsaturated ester (20) & SI-55 \\
\hline DEPT 90/135 (75 MHz, $\left.\mathrm{CDCl}_{3}\right)$ - Unsaturated ester (20) & SI-56 \\
\hline IR (Film) - Unsaturated ester (20) & SI-56 \\
\hline${ }^{1} \mathrm{H}$ NMR (300 MHz, $\mathrm{CDCl}_{3}$ ) - Allylic Alcohol from (20) & SI-57 \\
\hline${ }^{13} \mathrm{C}$ NMR $\left(75 \mathrm{MHz}, \mathrm{CDCl}_{3}\right.$ ) - Allylic Alcohol from (20) & SI-57 \\
\hline DEPT 90/135 (75 MHz, CDCl ${ }_{3}$ ) - Allylic Alcohol from (20) & SI-58 \\
\hline${ }^{1} \mathrm{H}$ NMR (300 MHz, $\mathrm{CDCl}_{3}$ ) - Bromide (21) & SI-58 \\
\hline${ }^{13} \mathrm{C} \mathrm{NMR}\left(75 \mathrm{MHz}, \mathrm{CDCl}_{3}\right)$ - Bromide (21) & SI-59 \\
\hline DEPT 90/135 (75 MHz, $\left.\mathrm{CDCl}_{3}\right)$ - Bromide (21) & SI-59 \\
\hline IR (Film) - Bromide (21) & SI-60 \\
\hline${ }^{1} \mathrm{H}$ NMR (300 MHz, $\mathrm{CDCl}_{3}$ ) - Diene (22) & SI-61 \\
\hline${ }^{13} \mathrm{C} \mathrm{NMR} \mathrm{(75} \mathrm{MHz,} \mathrm{CDCl}_{3}$ ) - Diene (22) & SI-62 \\
\hline DEPT 90/135 (75 MHz, CDCl ${ }_{3}$ ) - Diene (22) & SI-62 \\
\hline${ }^{1}$ H NMR (300 MHz, $\mathrm{CDCl}_{3}$ ) - Alkyl iodide (2) & SI-63 \\
\hline${ }^{13} \mathrm{C}$ NMR (75 MHz, $\mathrm{CDCl}_{3}$ ) - Alkyl iodide (2) & SI-63 \\
\hline
\end{tabular}




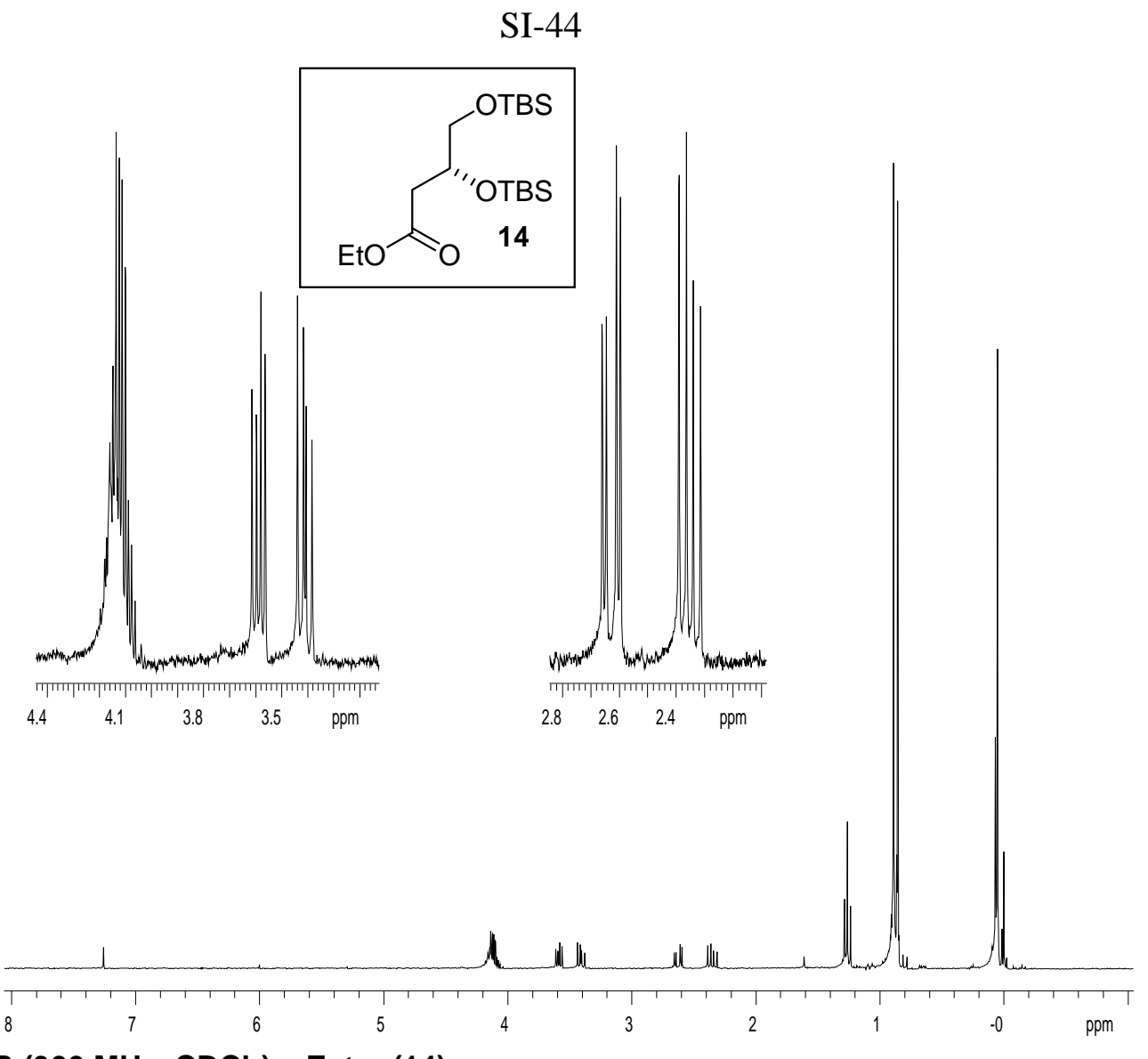

${ }^{1} \mathrm{H}$ NMR (300 MHz, $\mathrm{CDCl}_{3}$ ) - Ester (14)

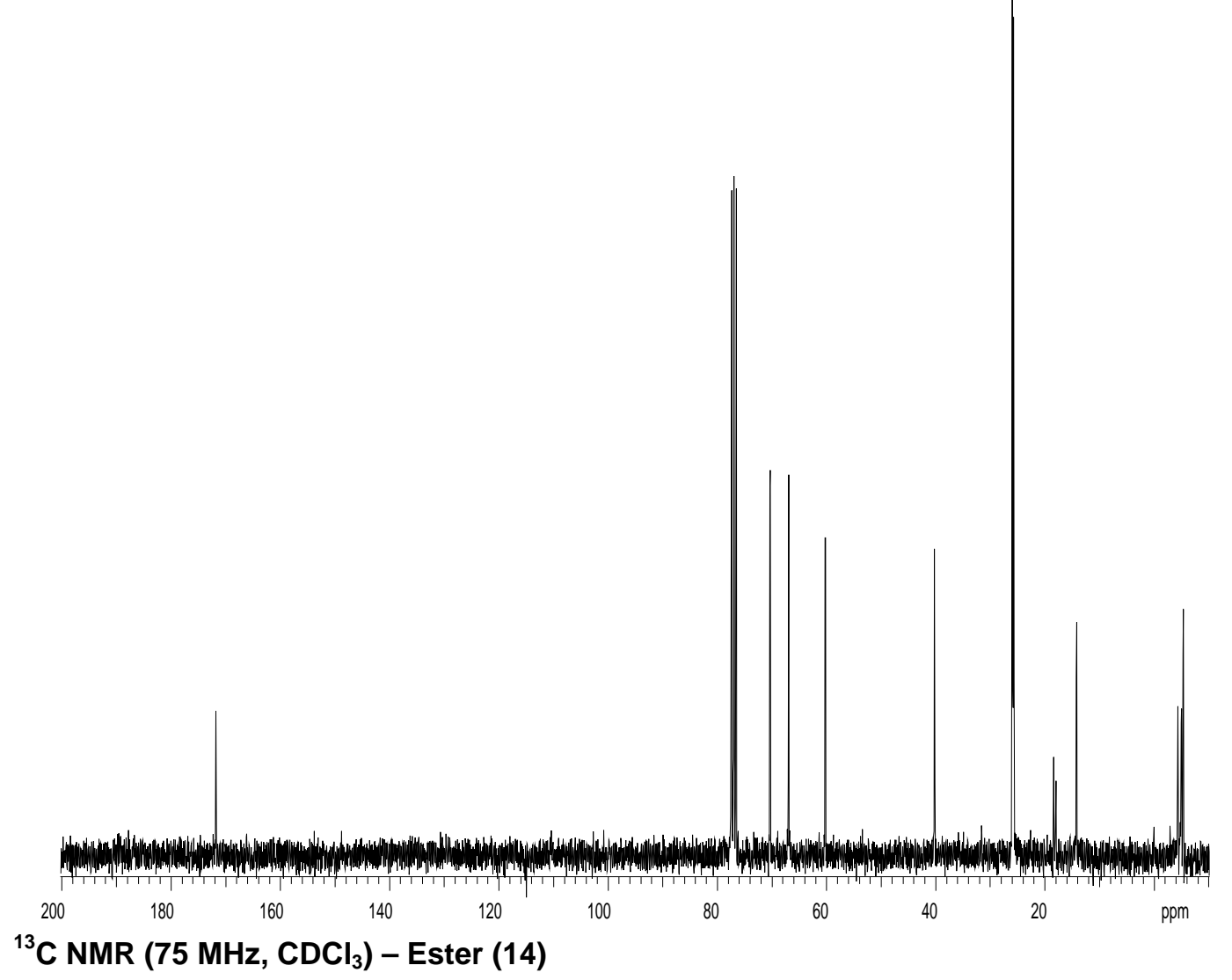



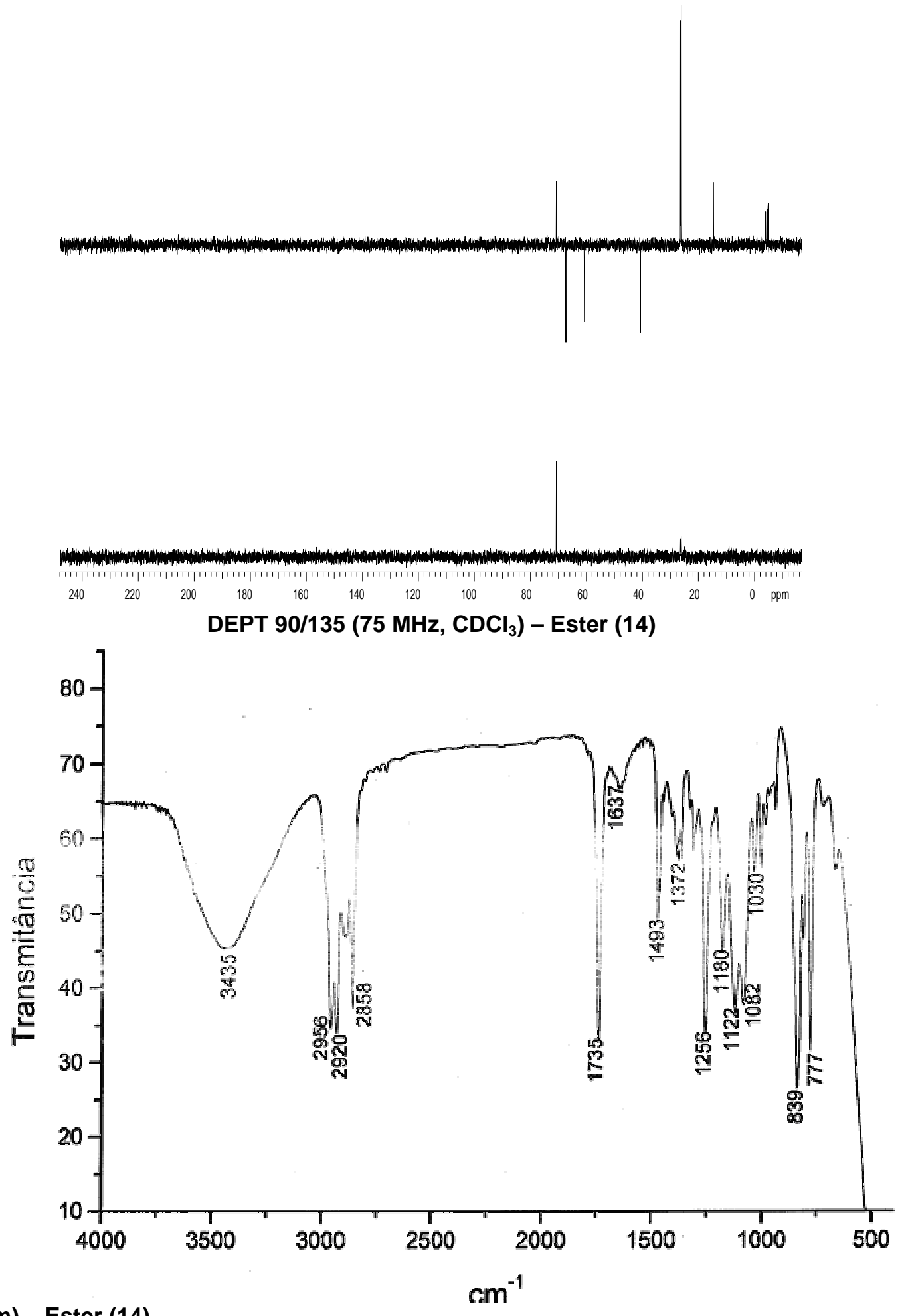

IR (film) - Ester (14) 


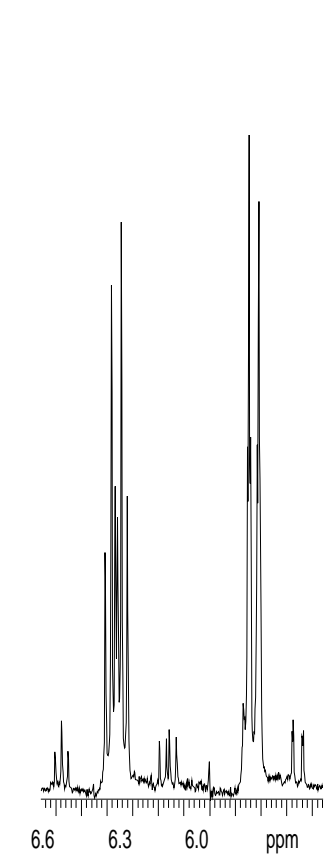

SI-46
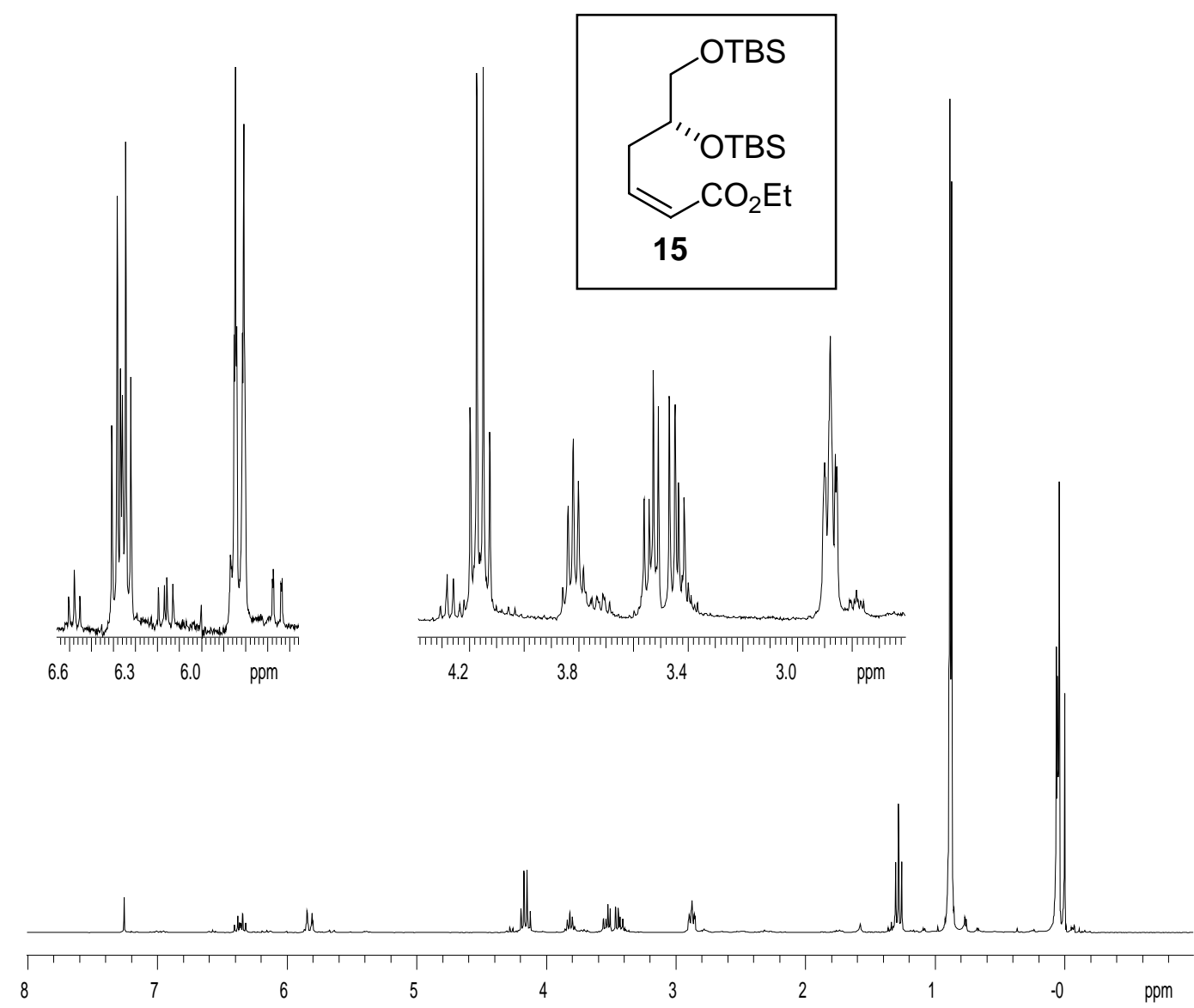

${ }^{1} \mathrm{H}$ NMR (300 MHz, $\mathrm{CDCl}_{3}$ ) - Ester (15) 


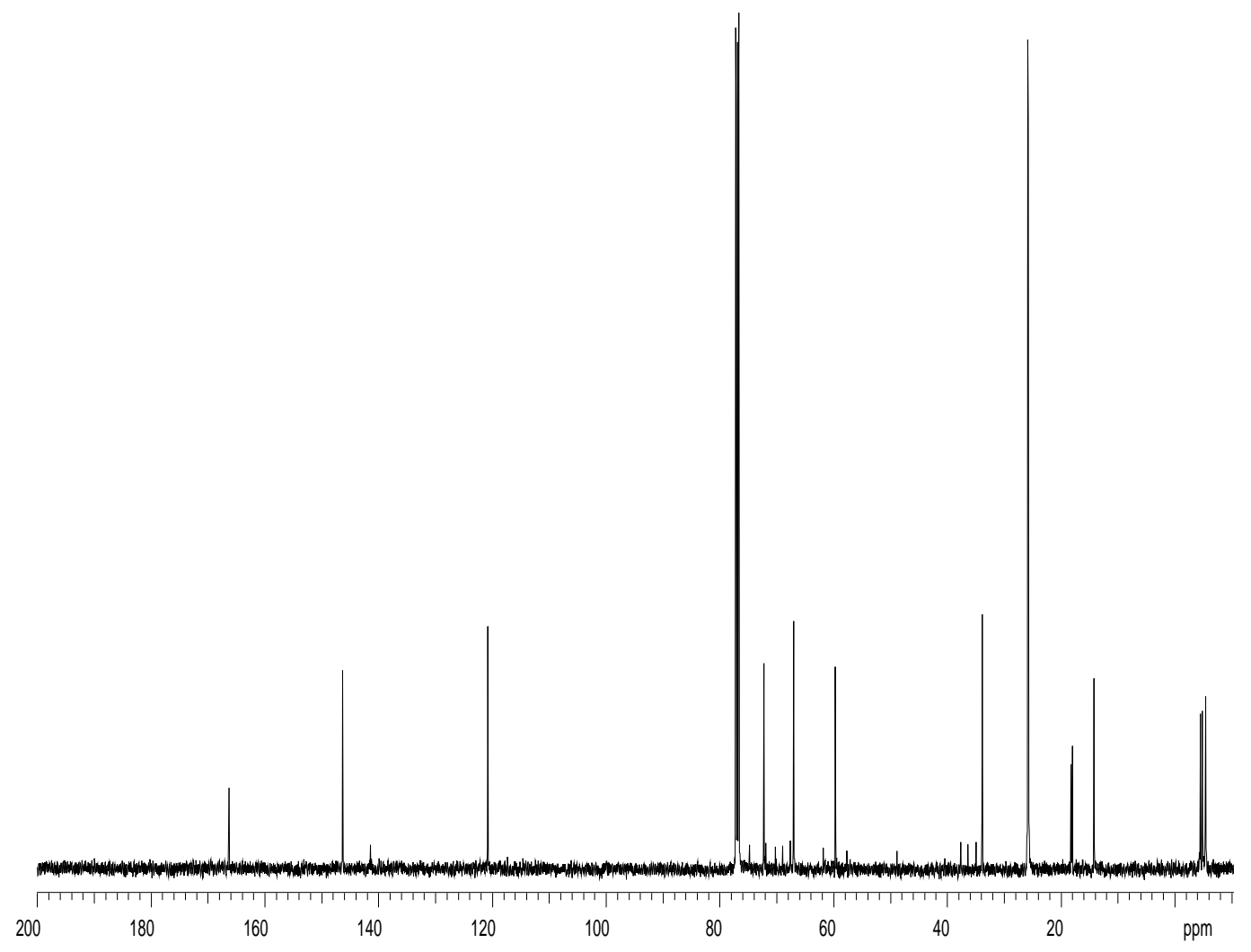

${ }^{13} \mathrm{C}$ NMR (75 MHz, $\mathrm{CDCl}_{3}$ ) - Ester (15)
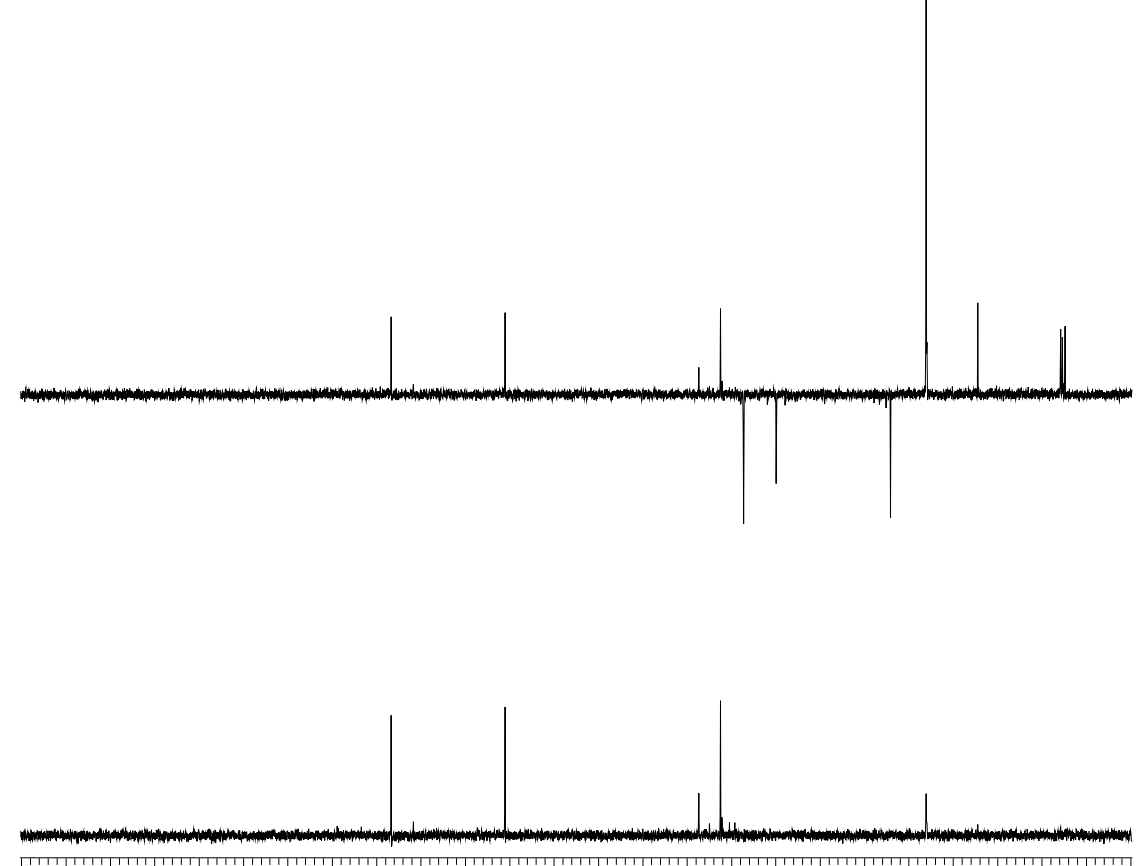

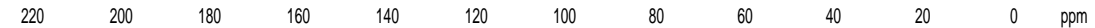

DEPT 90/135 (75 MHz, $\mathrm{CDCl}_{3}$ ) - Ester (15) 


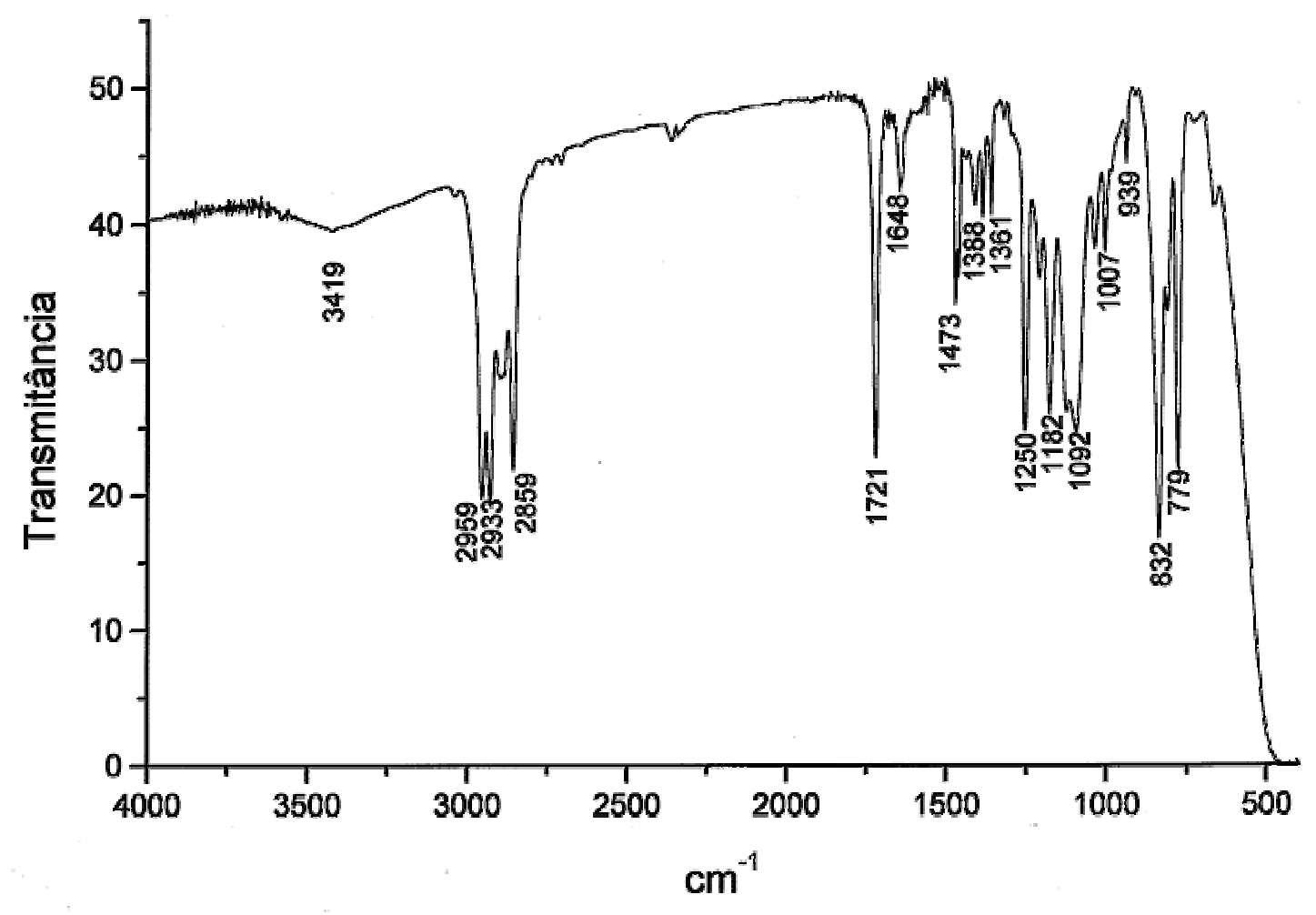

IR (film) - Ester (15) 


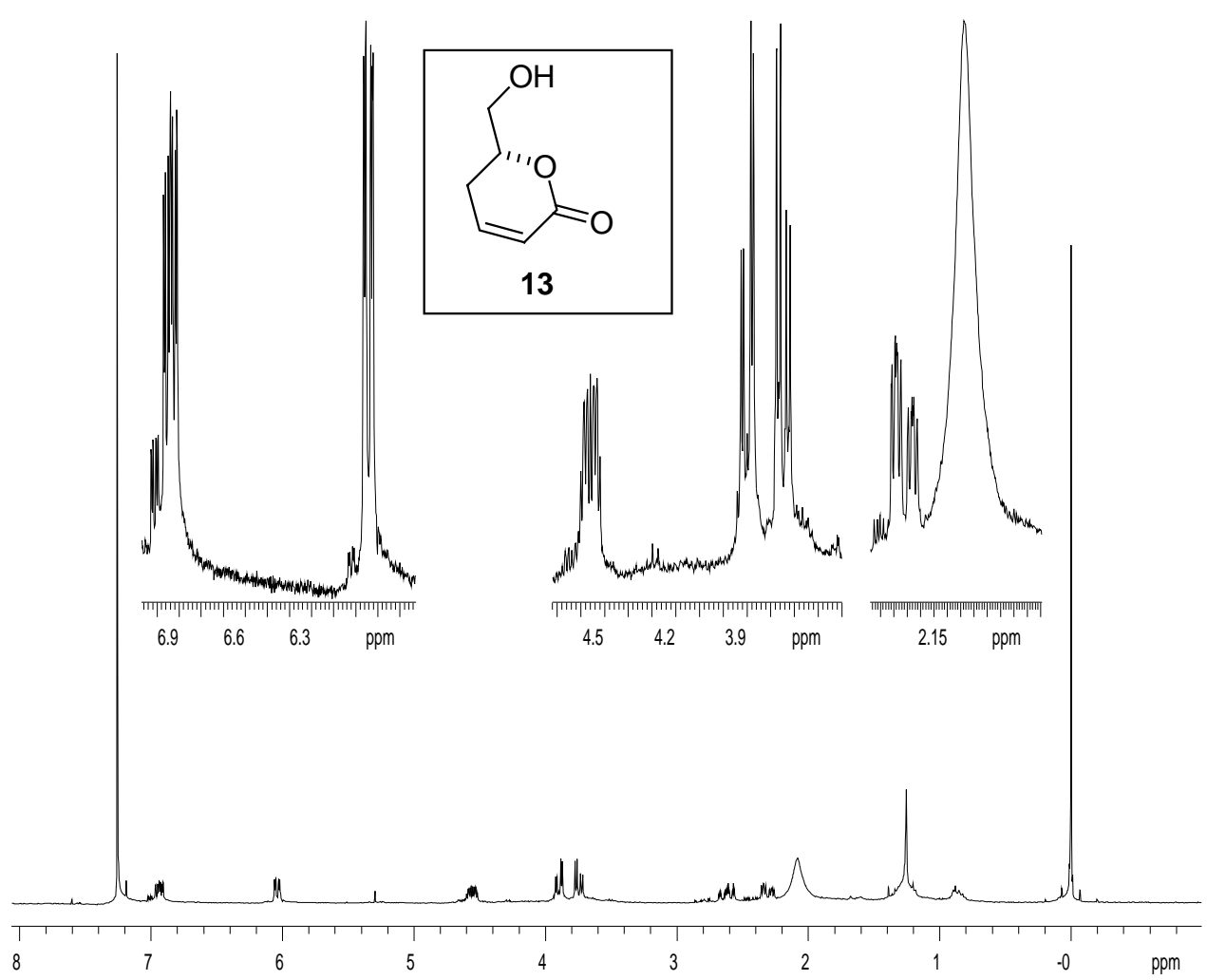

${ }^{1} \mathrm{H}$ NMR (300 MHz, $\mathrm{CDCl}_{3}$ ) - Lactone (13)

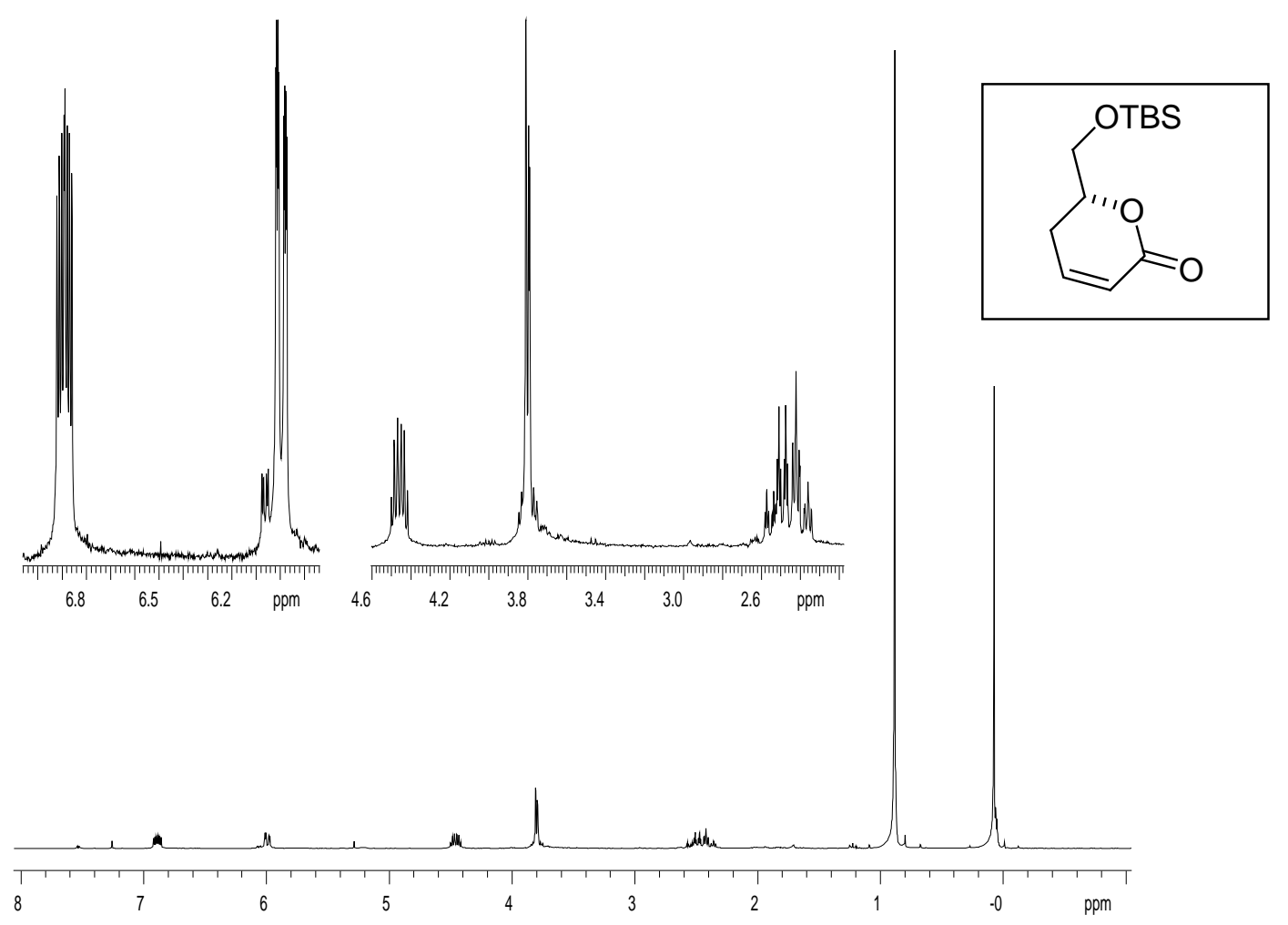

${ }^{1} \mathrm{H}$ NMR (300 MHz, $\mathrm{CDCl}_{3}$ ) - TBS-Lactone from (13) 
SI-50

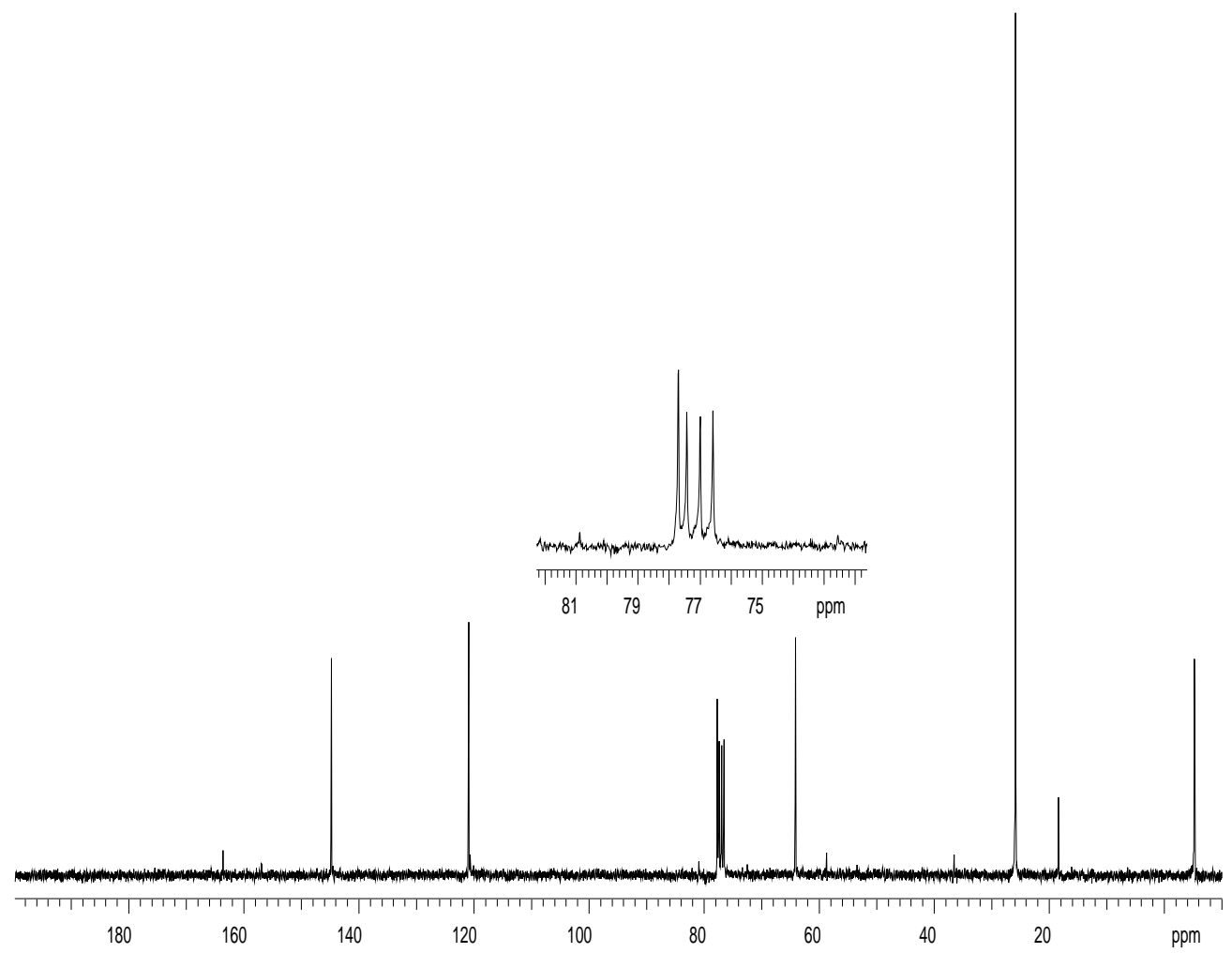

${ }^{13} \mathrm{C}$ NMR (75 MHz, $\mathrm{CDCl}_{3}$ ) - TBS-Lactone from (13)

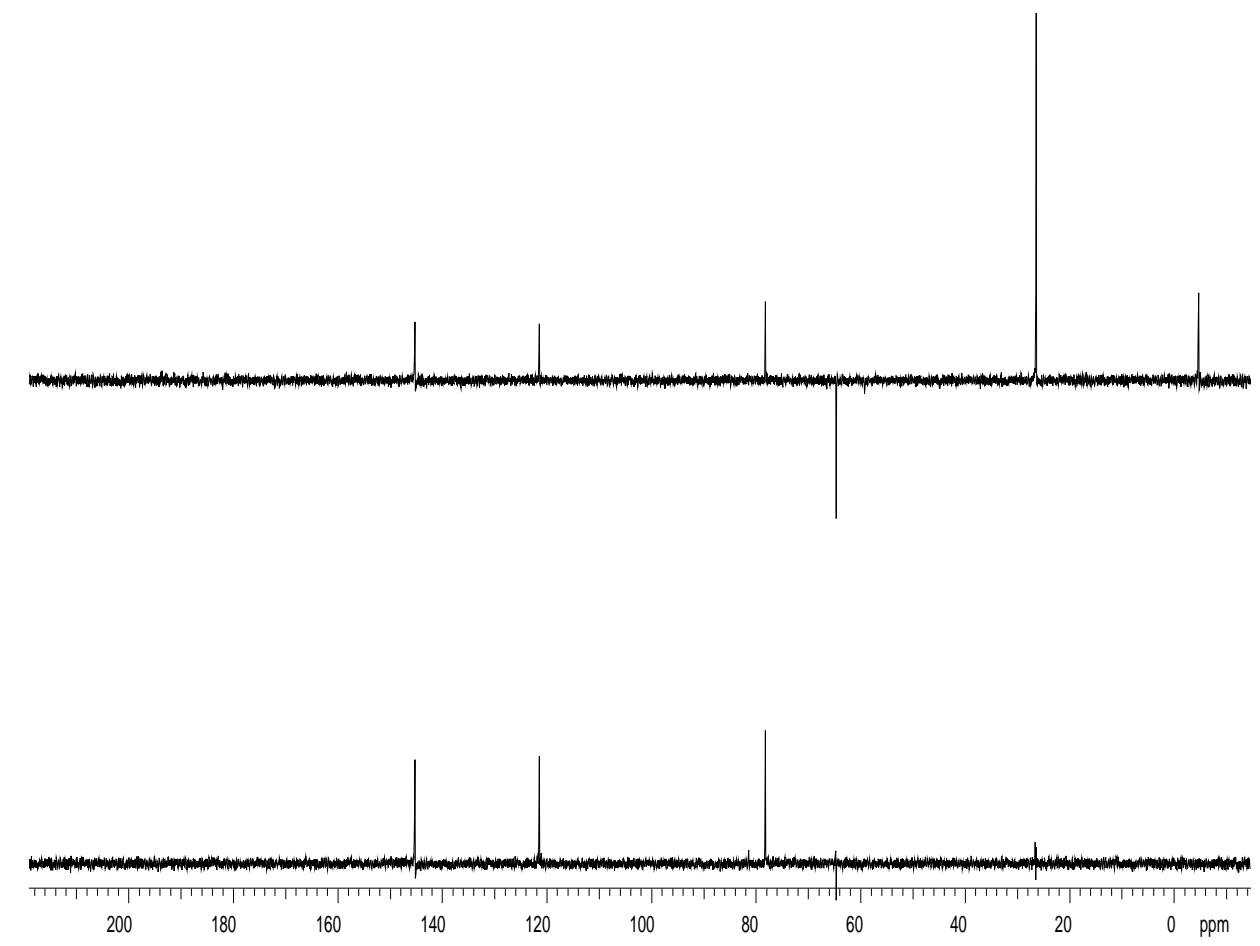

DEPT 90/135 (75 MHz, $\mathrm{CDCl}_{3}$ ) - TBS-Lactone from (13) 


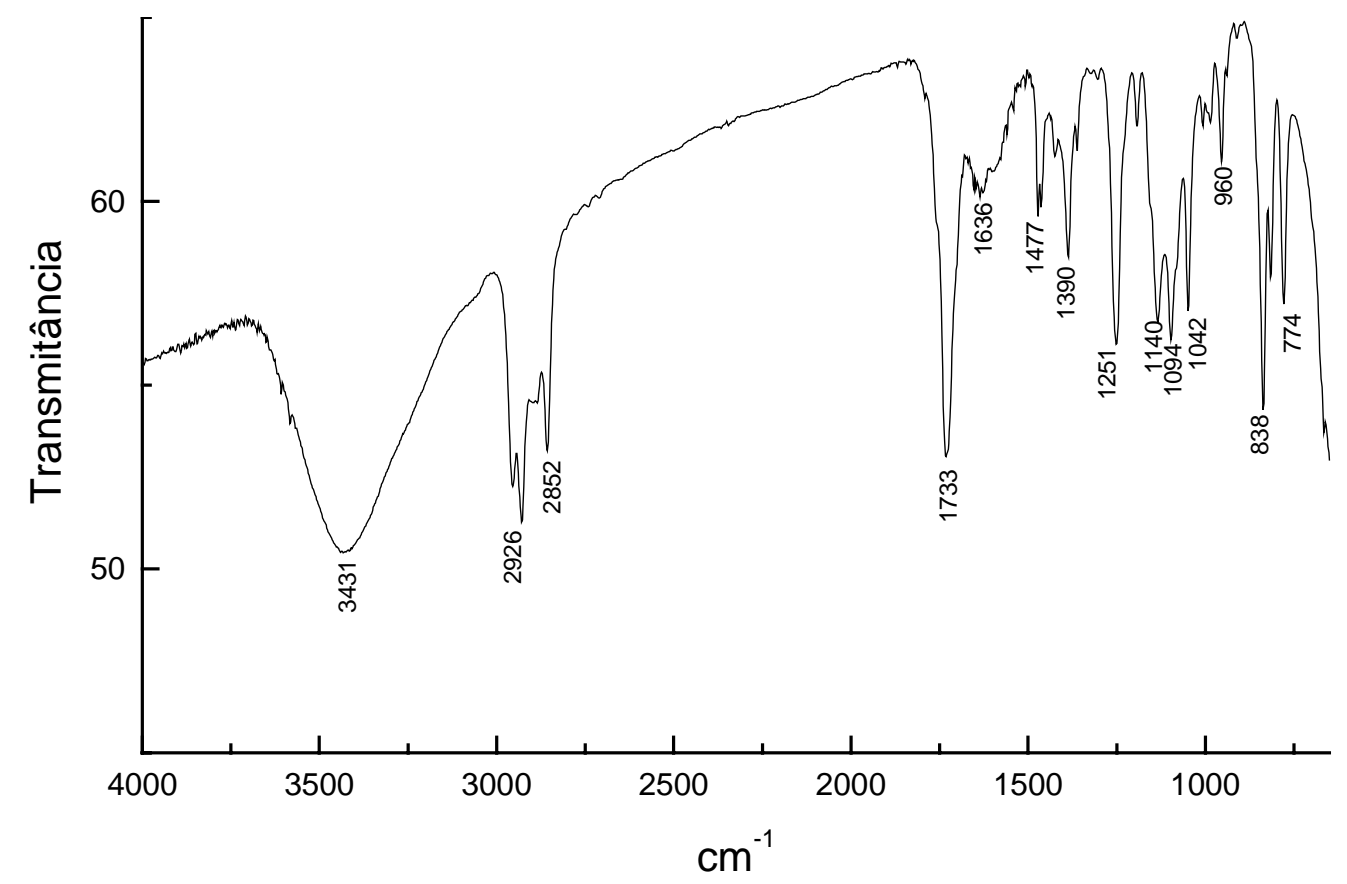

IR (Film) - TBS-Lactone from (13)
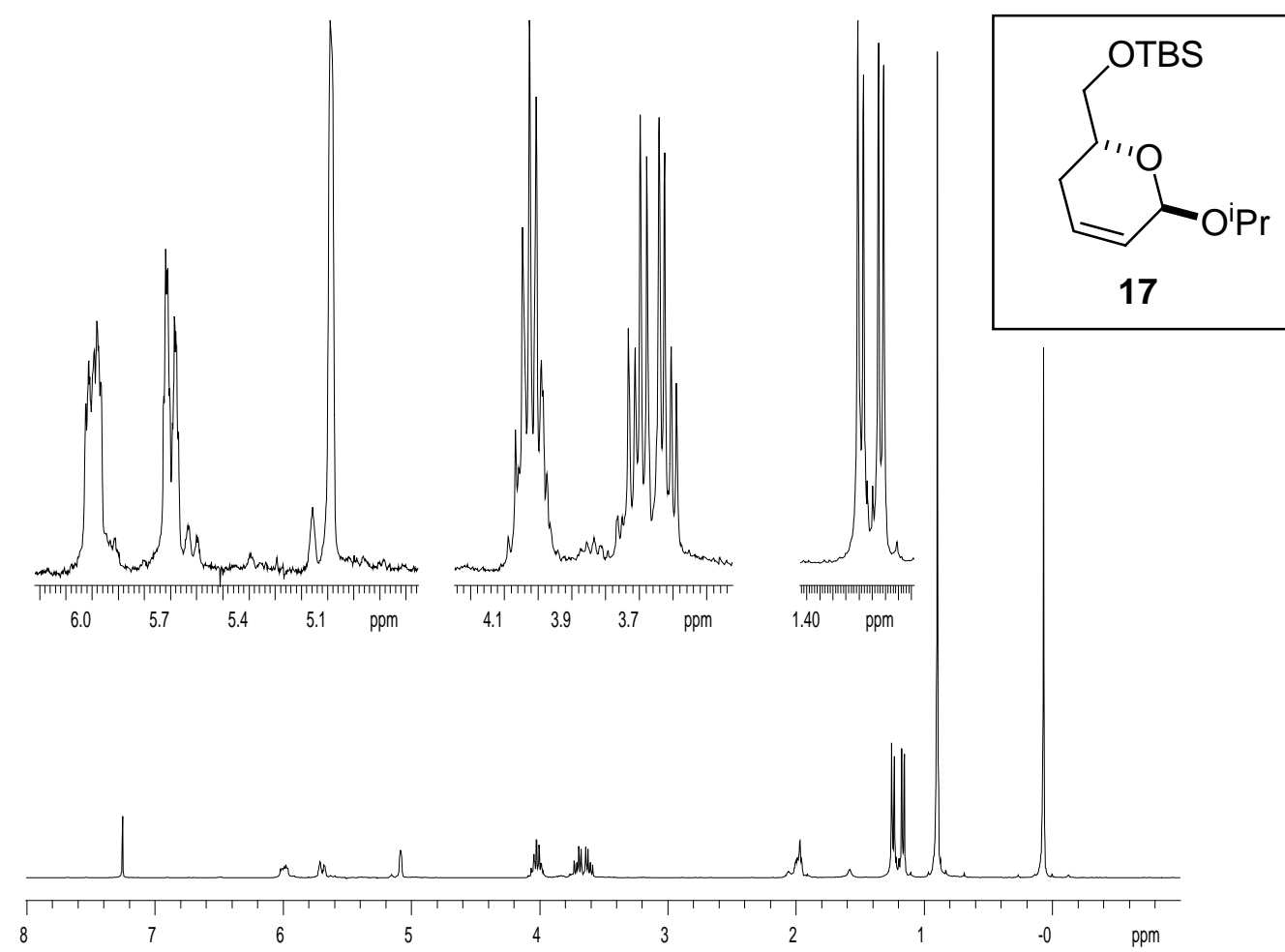

${ }^{1} \mathrm{H}$ NMR (300 MHz, $\mathrm{CDCl}_{3}$ ) - iPr-Lactol (17) 

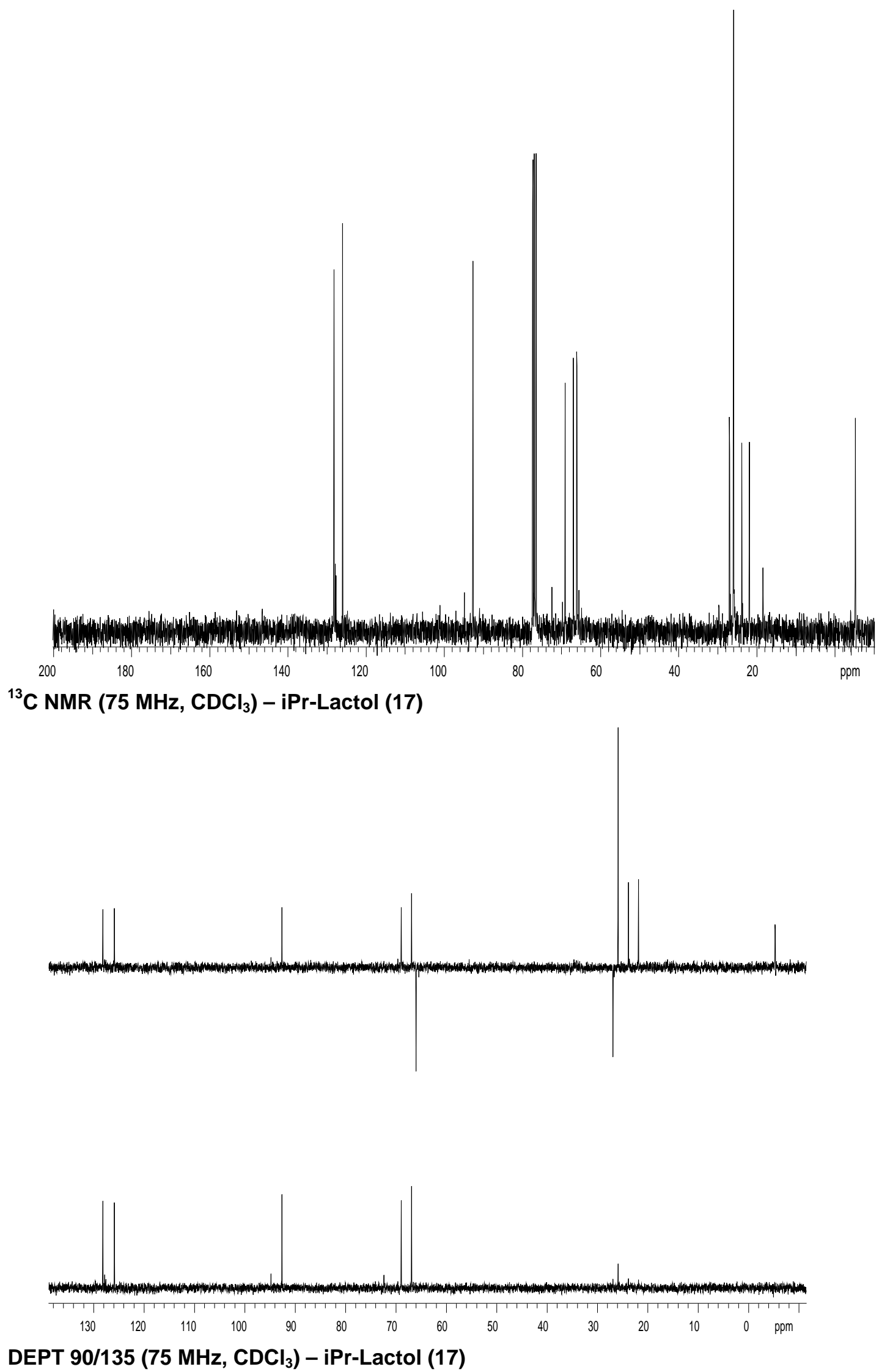
SI-53

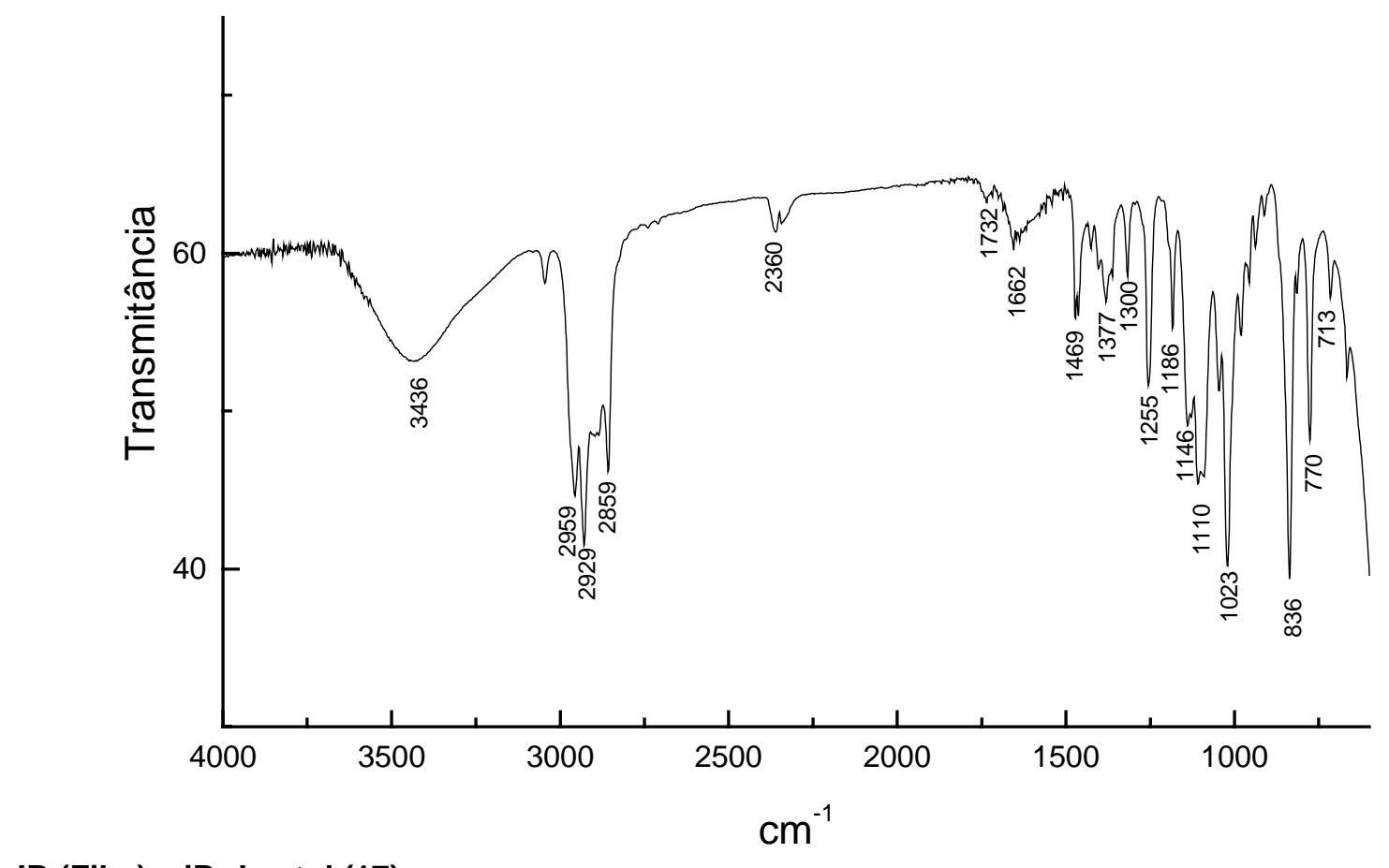

IR (Film) - iPr-Lactol (17)

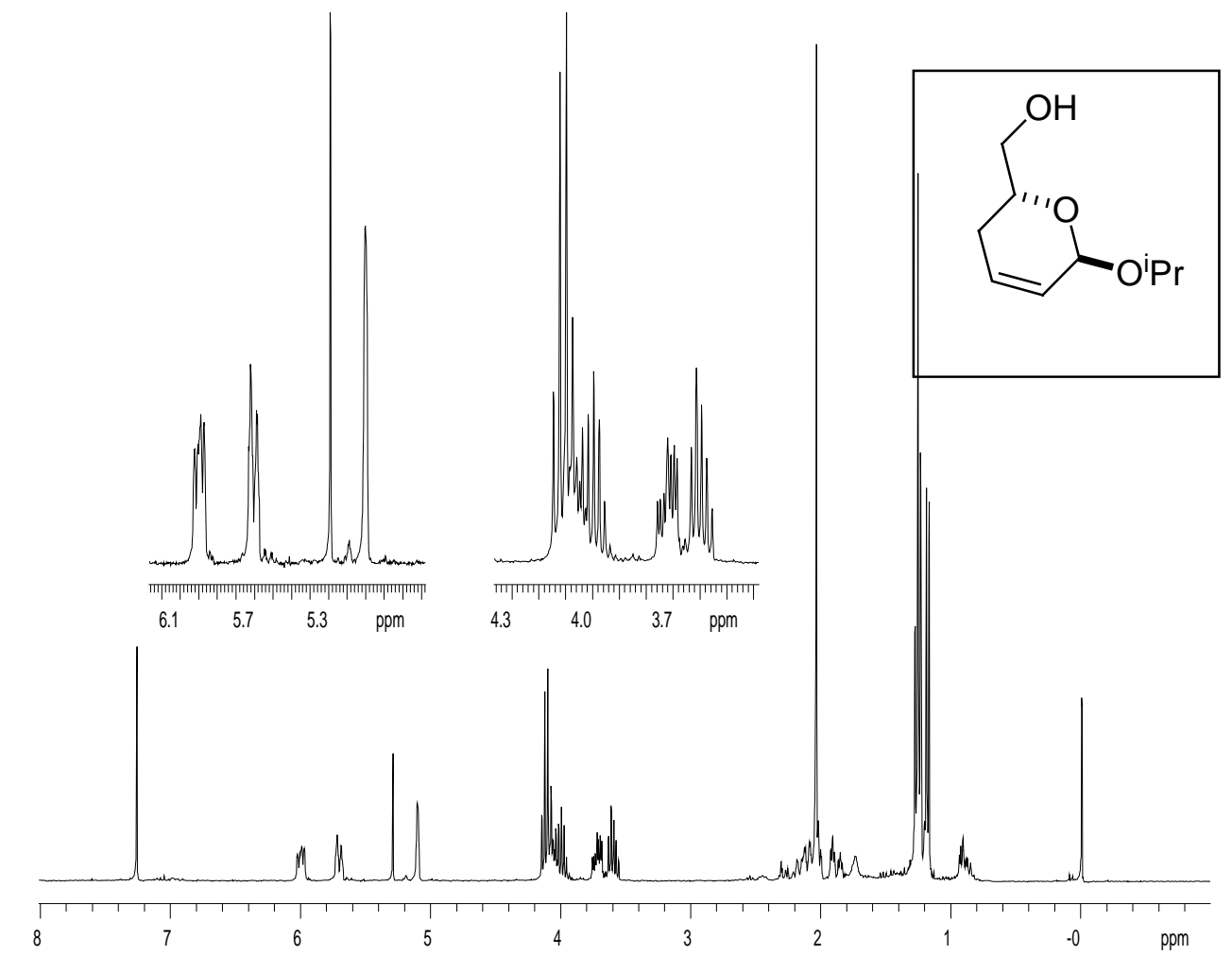

${ }^{1} \mathrm{H}$ NMR (300 MHz, $\mathrm{CDCl}_{3}$ ) - Alcohol from (17) 

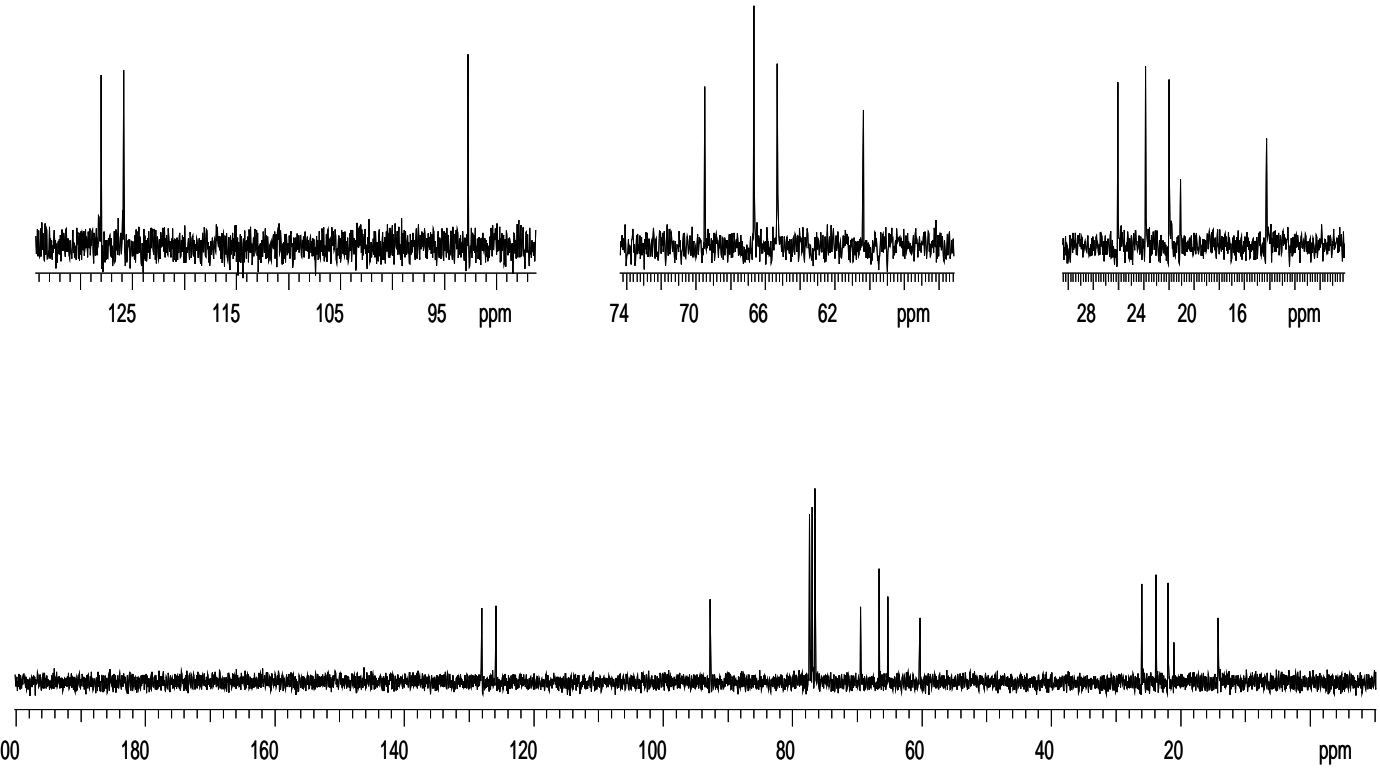

${ }^{13} \mathrm{C}$ NMR (75 MHz, $\mathrm{CDCl}_{3}$ ) - Alcohol from (17) 


\section{SI-55}
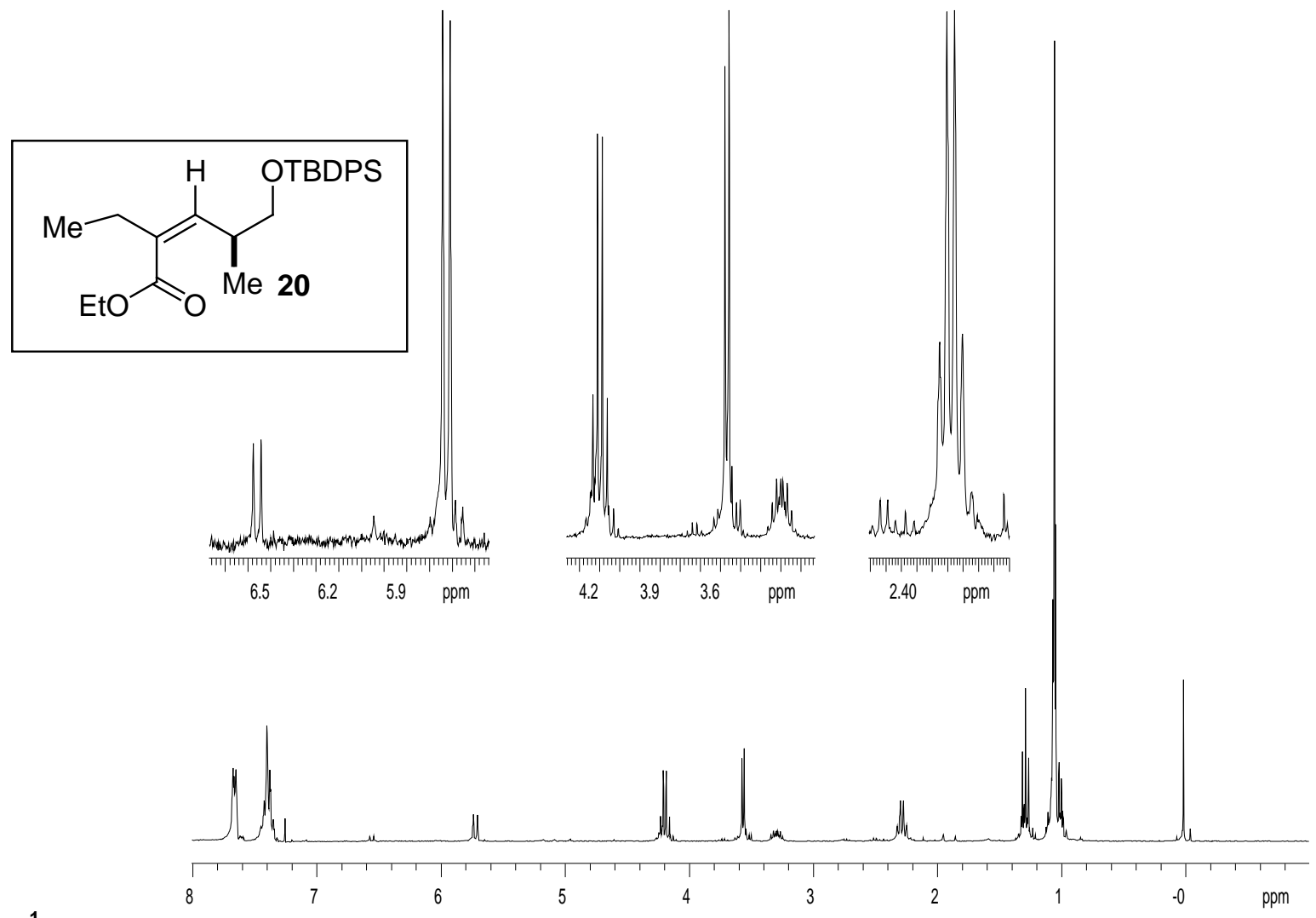

${ }^{1} \mathrm{H}$ NMR (300 MHz, $\mathrm{CDCl}_{3}$ ) - Unsaturated ester (20)

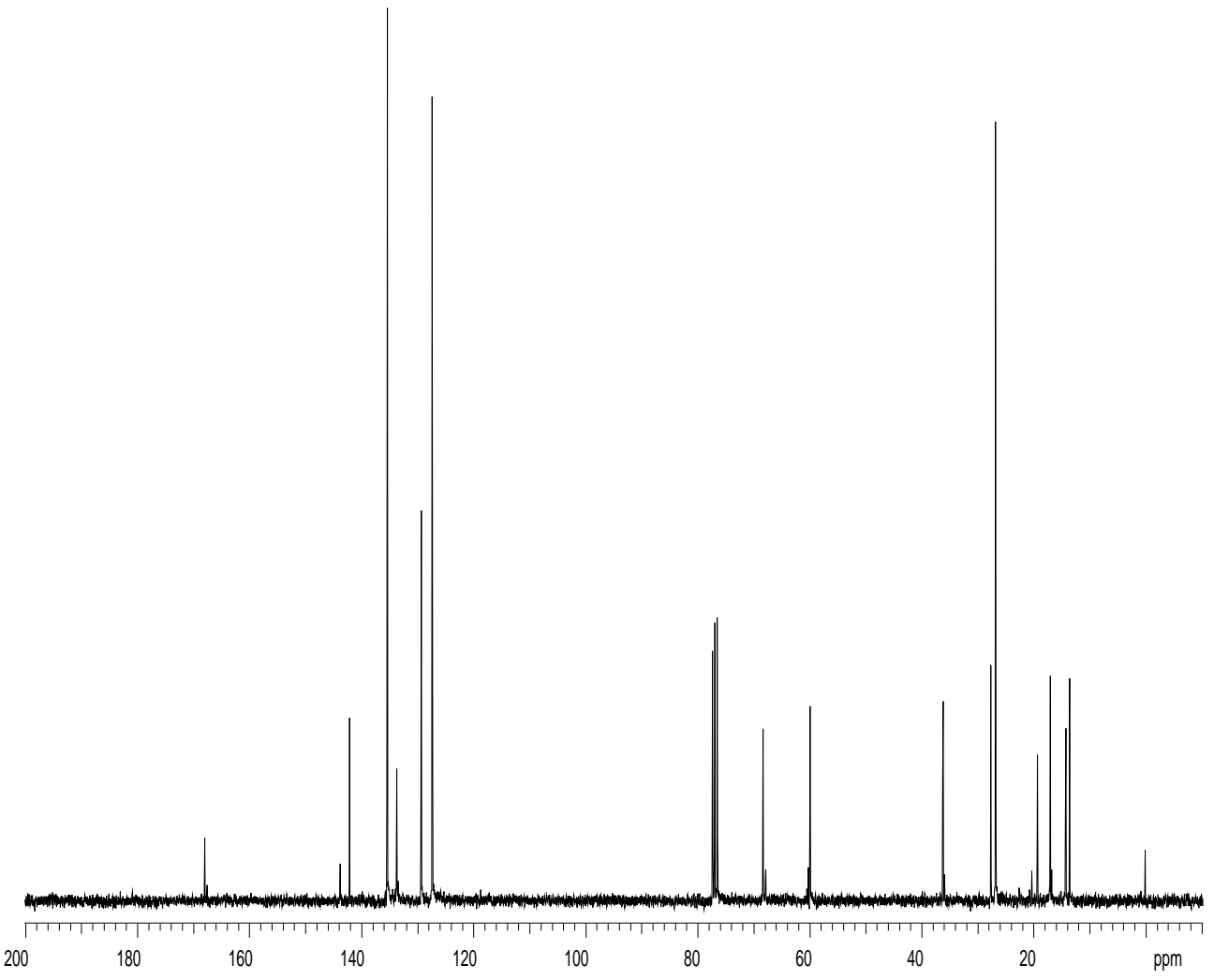

${ }^{13} \mathrm{C}$ NMR (75 MHz, $\mathrm{CDCl}_{3}$ ) - Unsaturated ester (20) 
SI-56
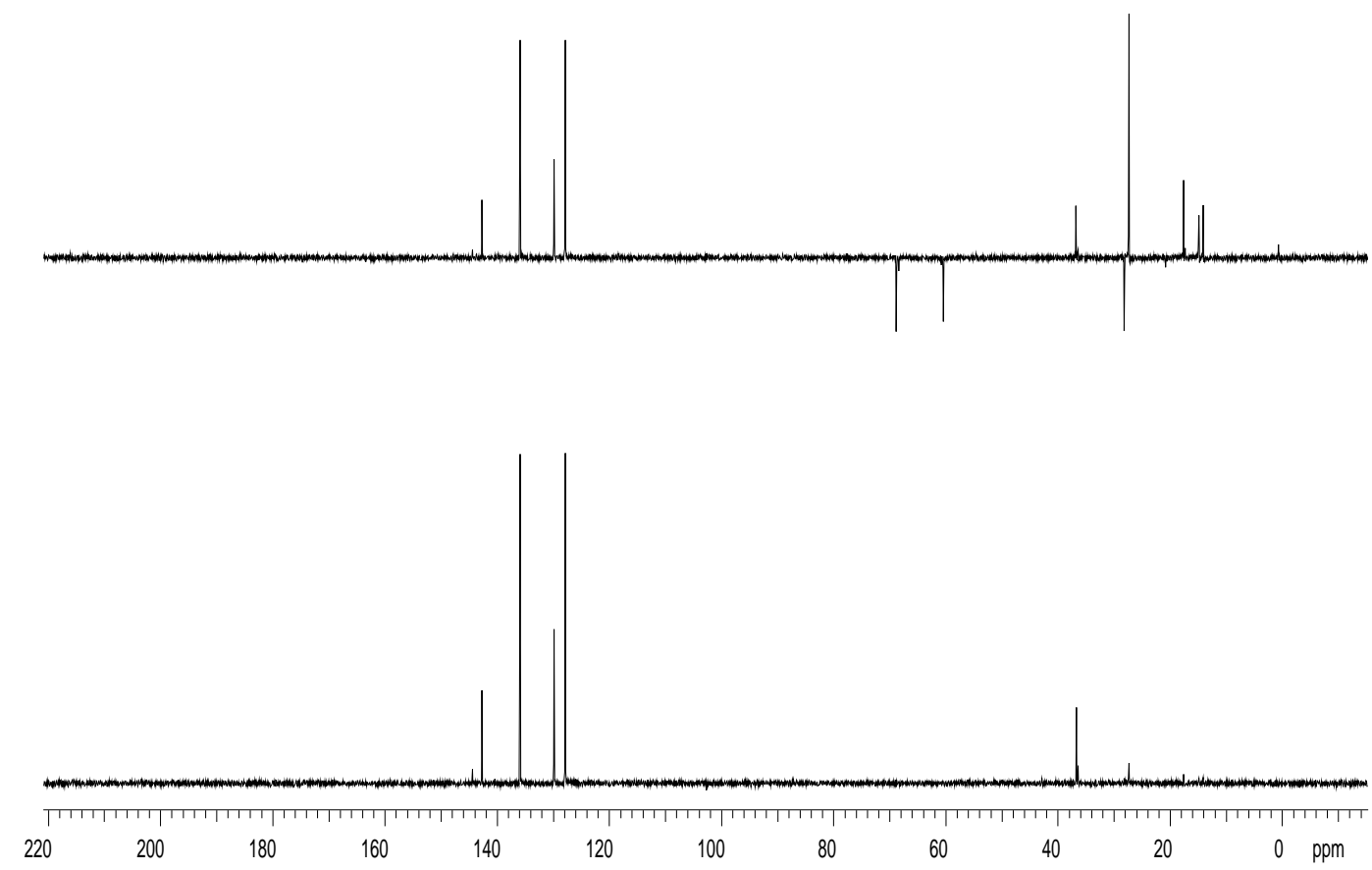

DEPT 90/135 (75 MHz, $\left.\mathrm{CDCl}_{3}\right)$ - Unsaturated ester (20)

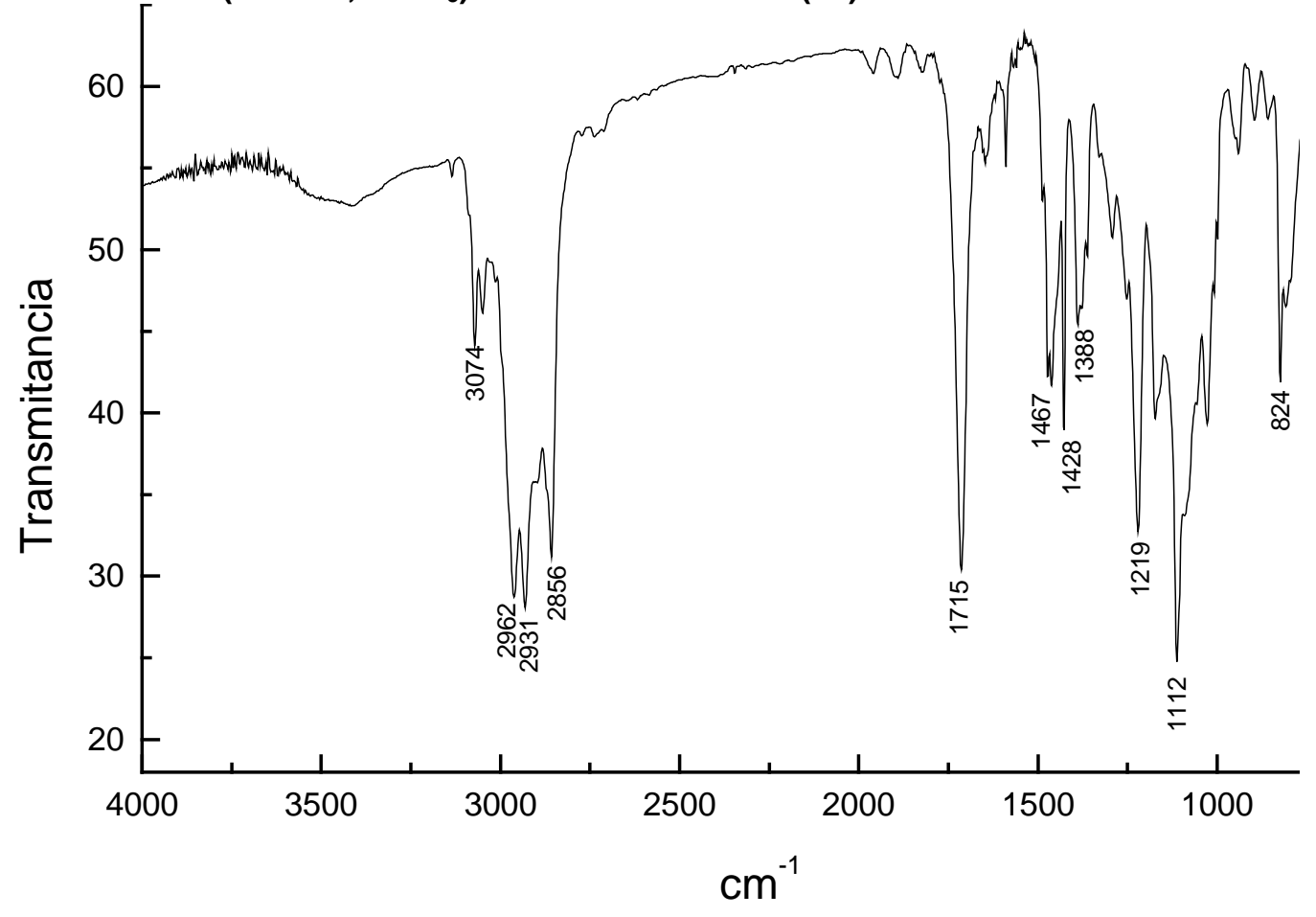

IR (Film) - Unsaturated ester (20) 


\section{SI-57}
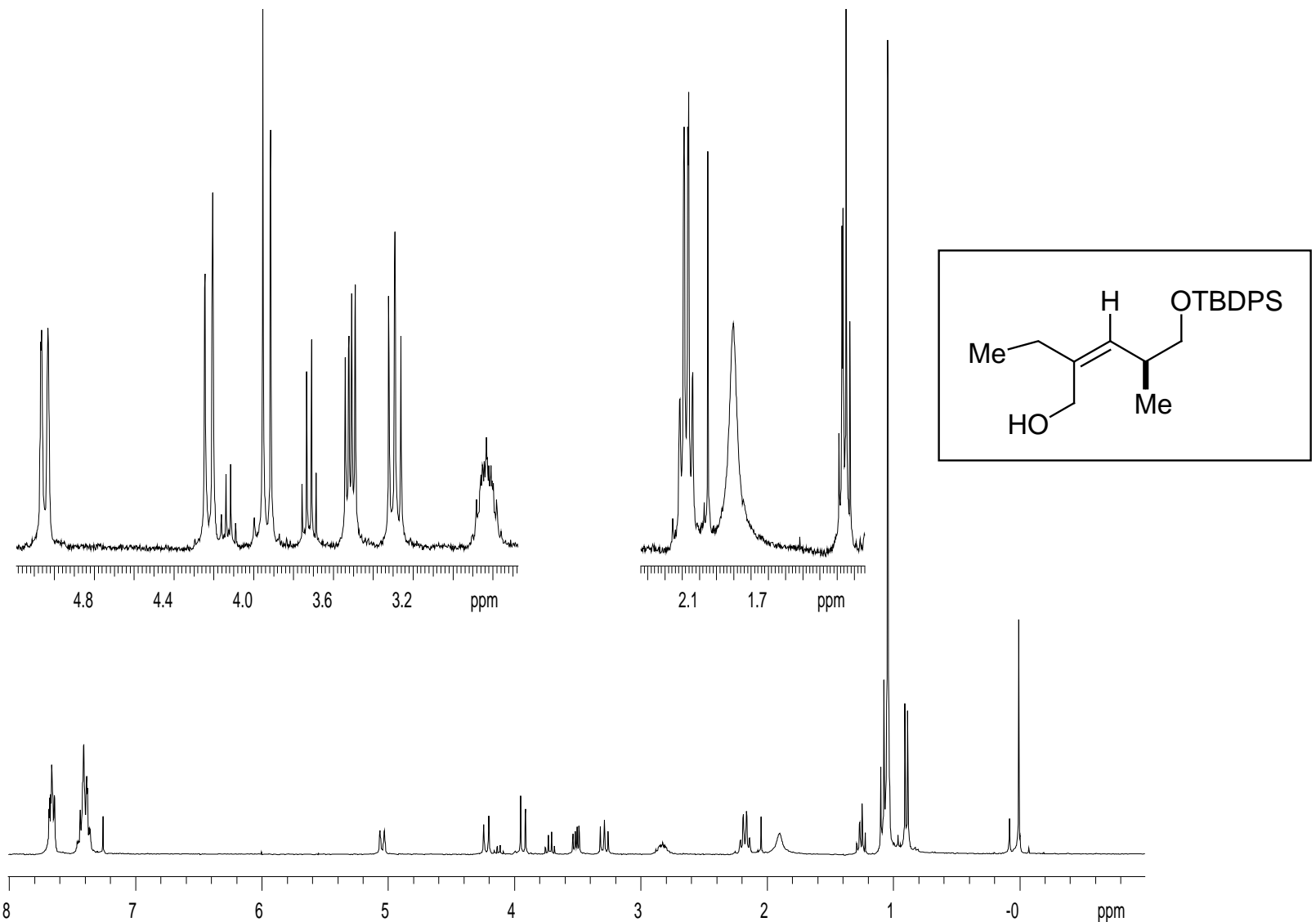

${ }^{1} \mathrm{H}$ NMR (300 MHz, $\mathrm{CDCl}_{3}$ ) - Allylic Alcohol from (20)

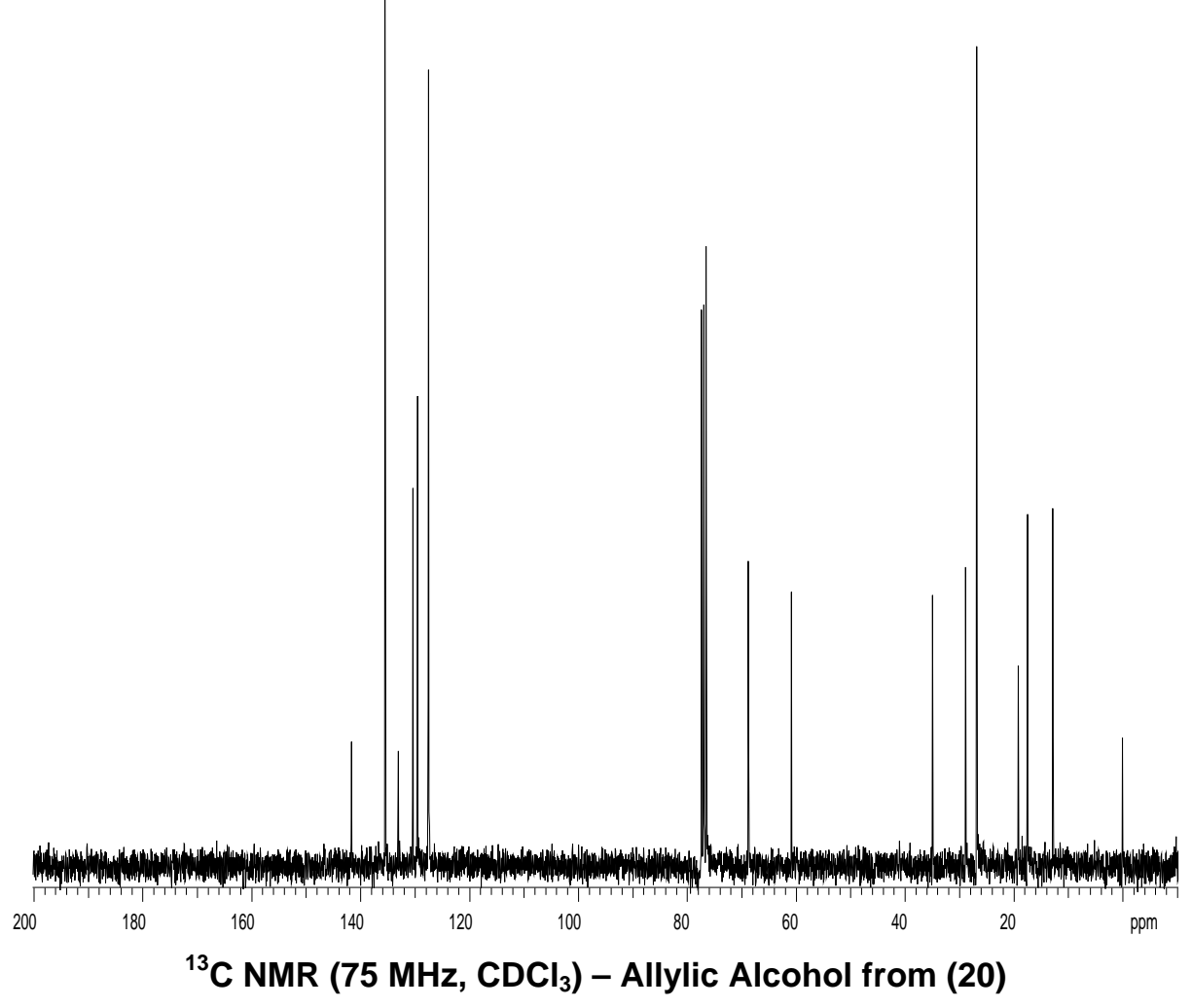


SI-58
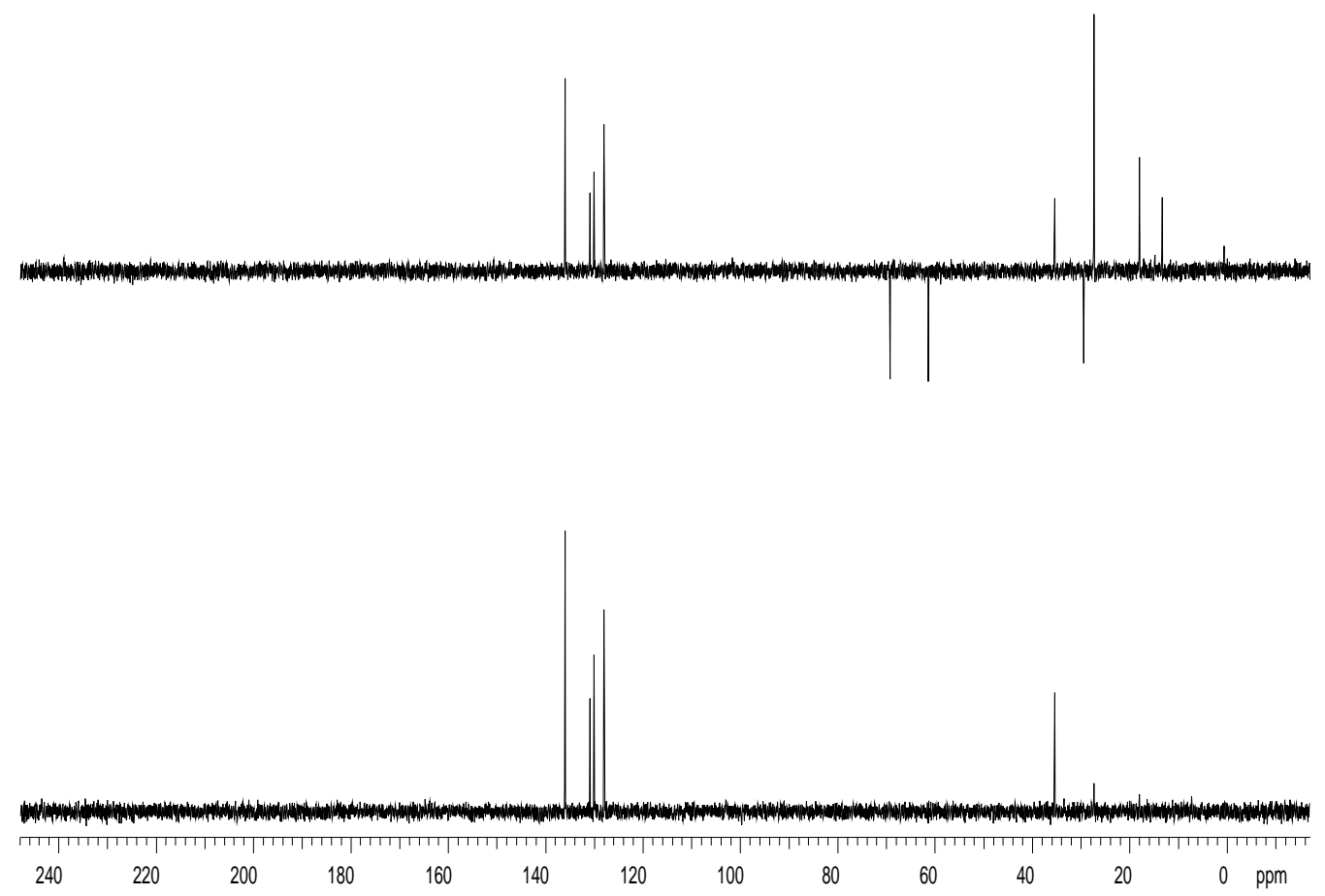

DEPT 90/135 (75 MHz, $\mathrm{CDCl}_{3}$ ) - Allylic Alcohol from (20)

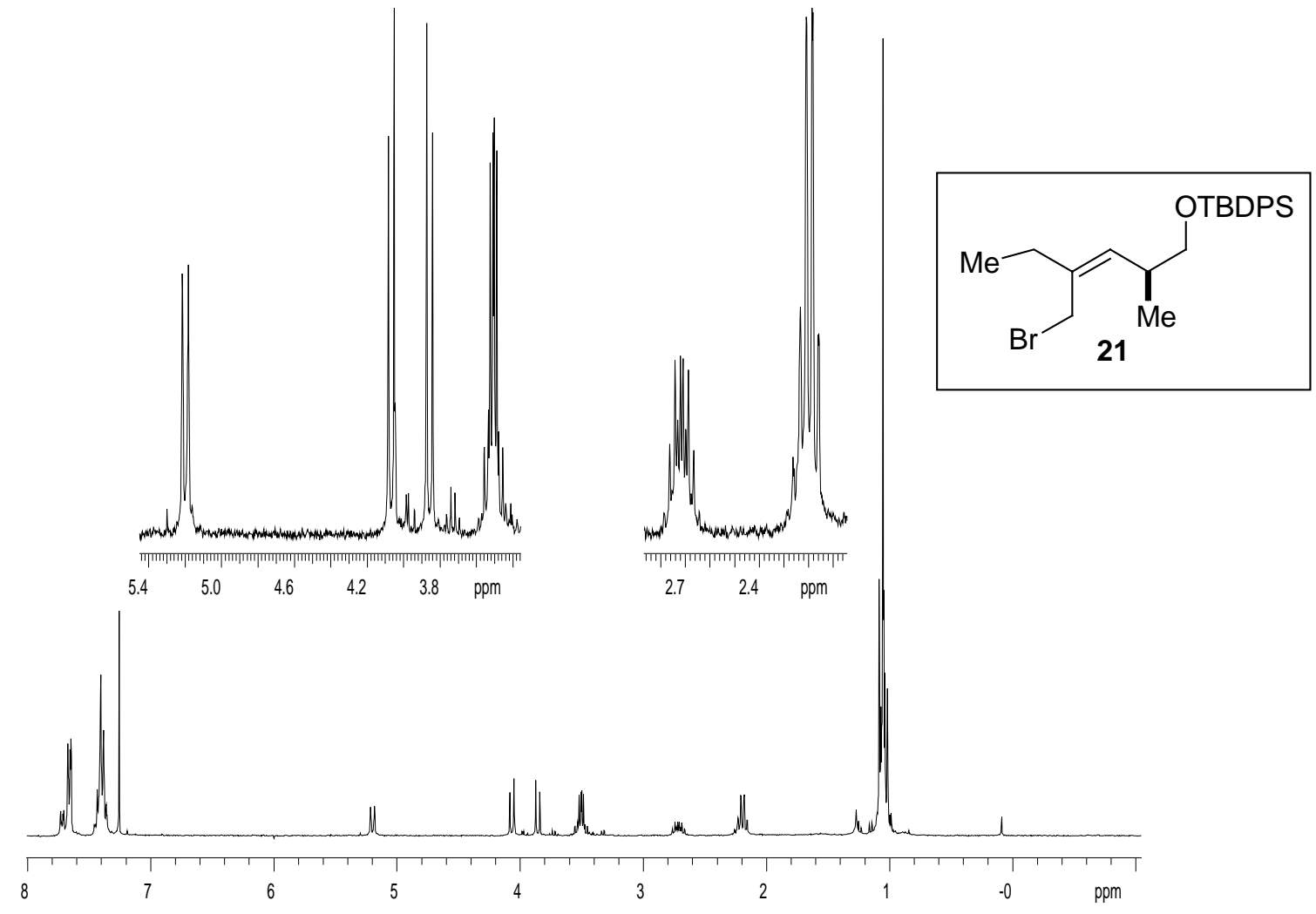

${ }^{1} \mathrm{H}$ NMR (300 MHz, $\mathrm{CDCl}_{3}$ ) - Bromide (21) 
SI-59
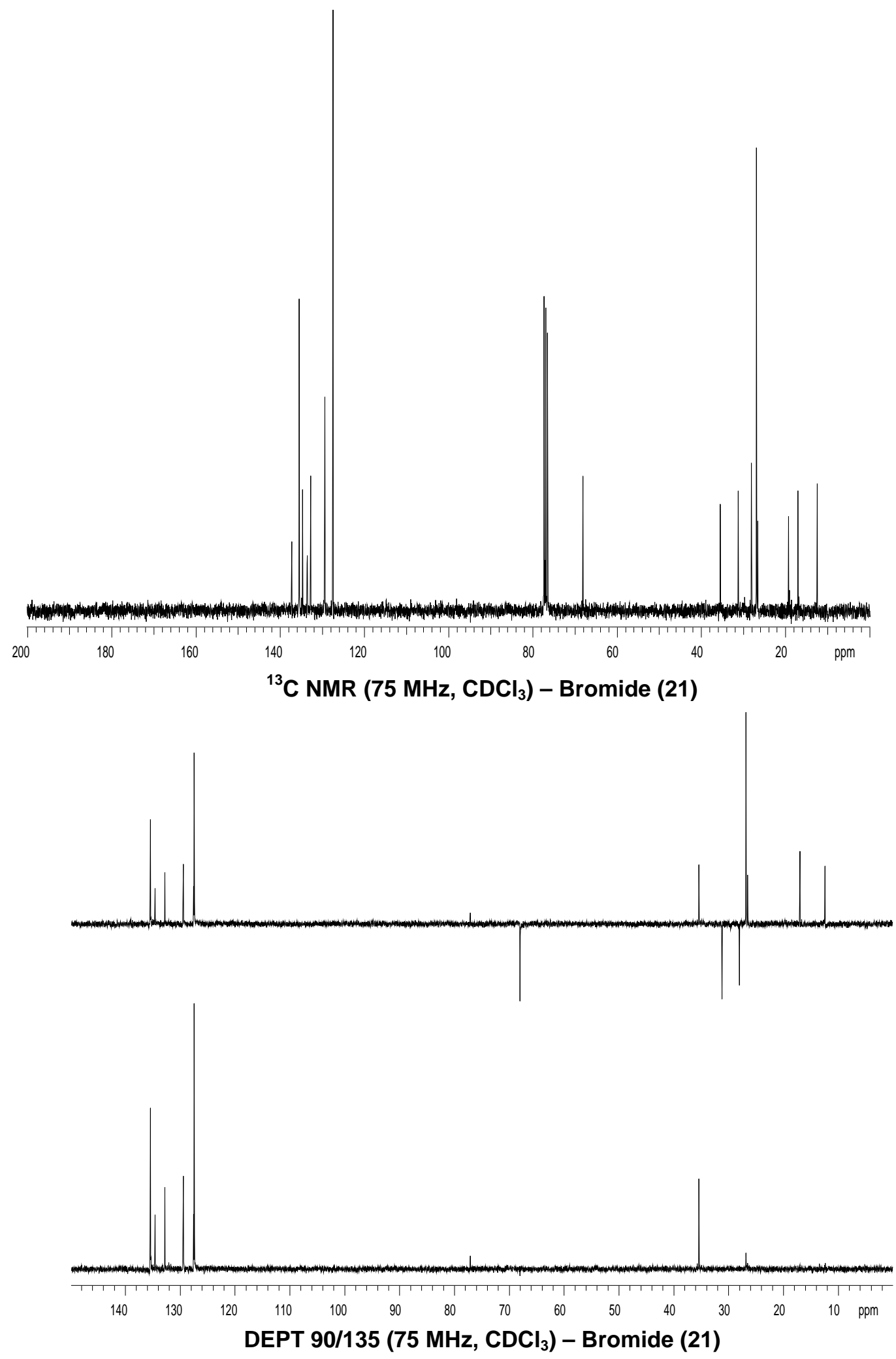
SI-60

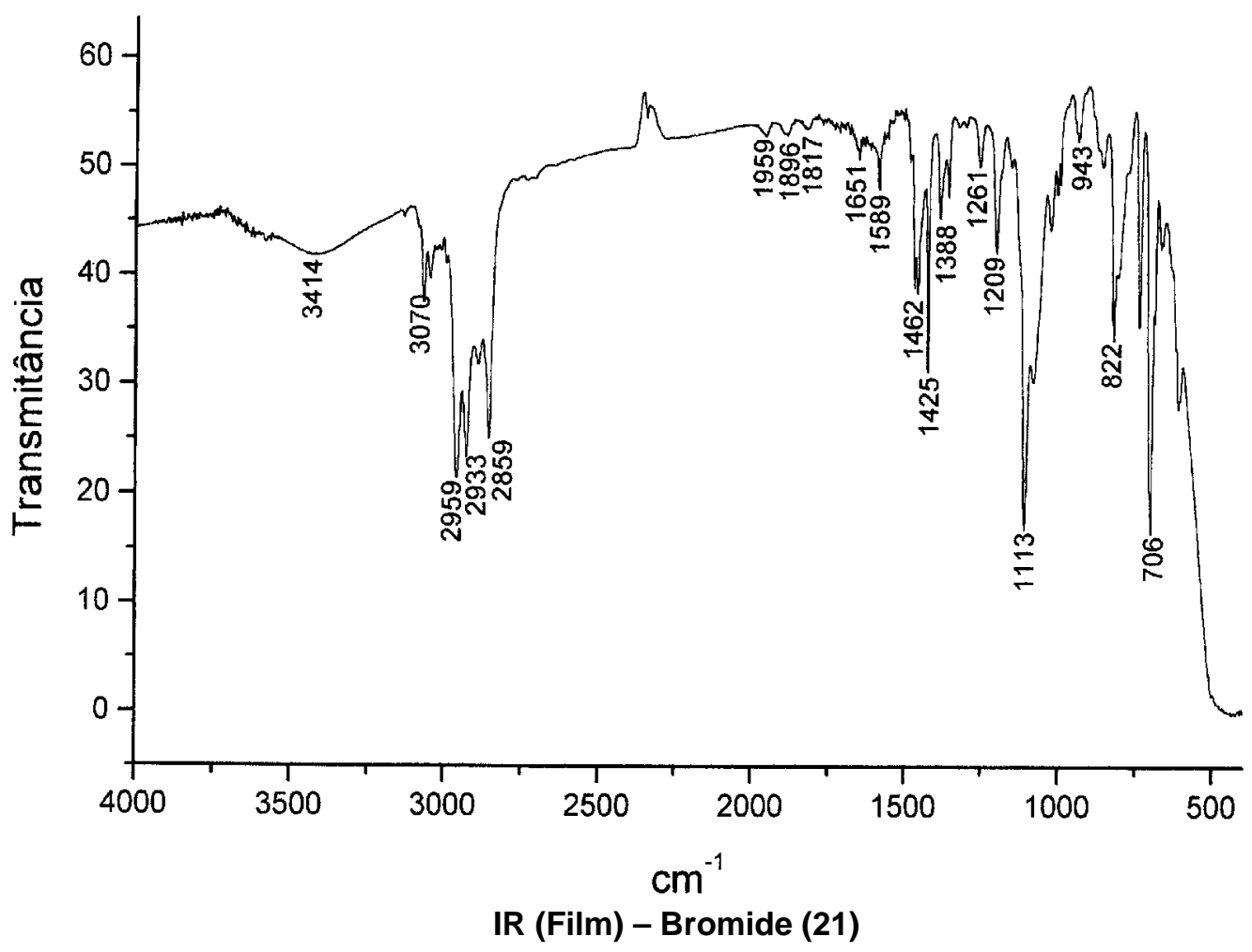




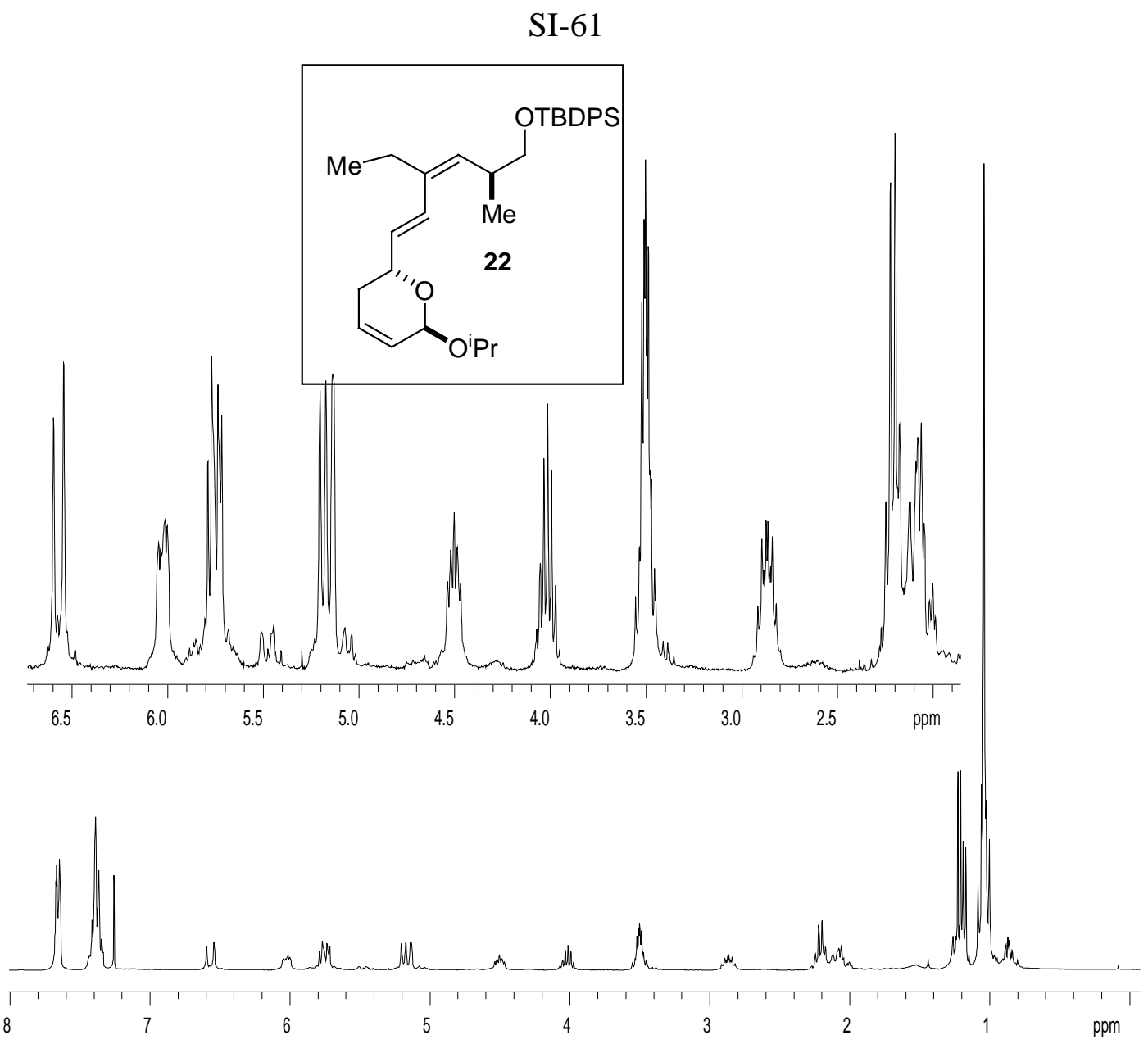

${ }^{1} \mathrm{H}$ NMR (300 MHz, $\mathrm{CDCl}_{3}$ ) - Diene (22) 


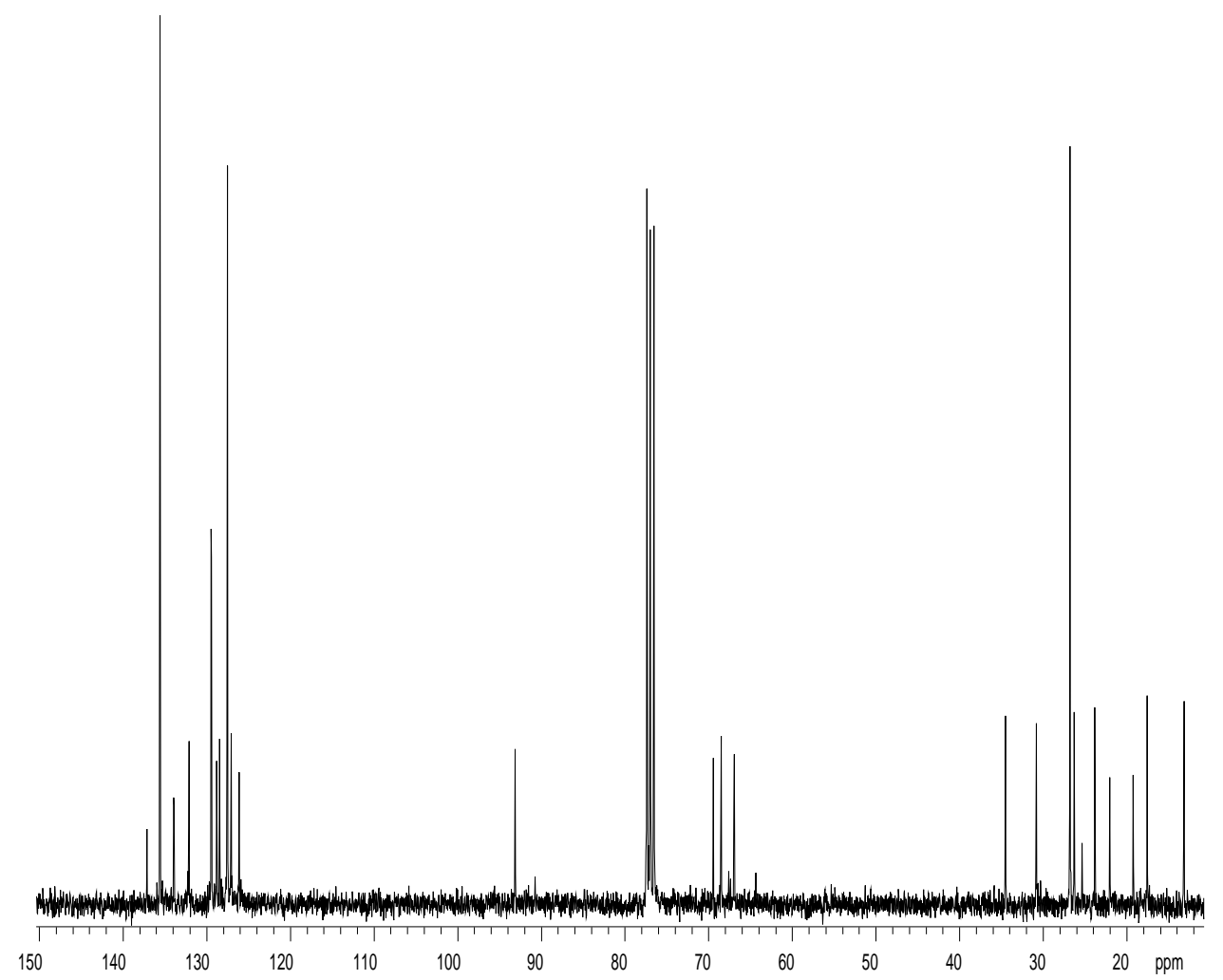

${ }^{13} \mathrm{C}$ NMR (75 MHz, $\mathrm{CDCl}_{3}$ ) - Diene (22)
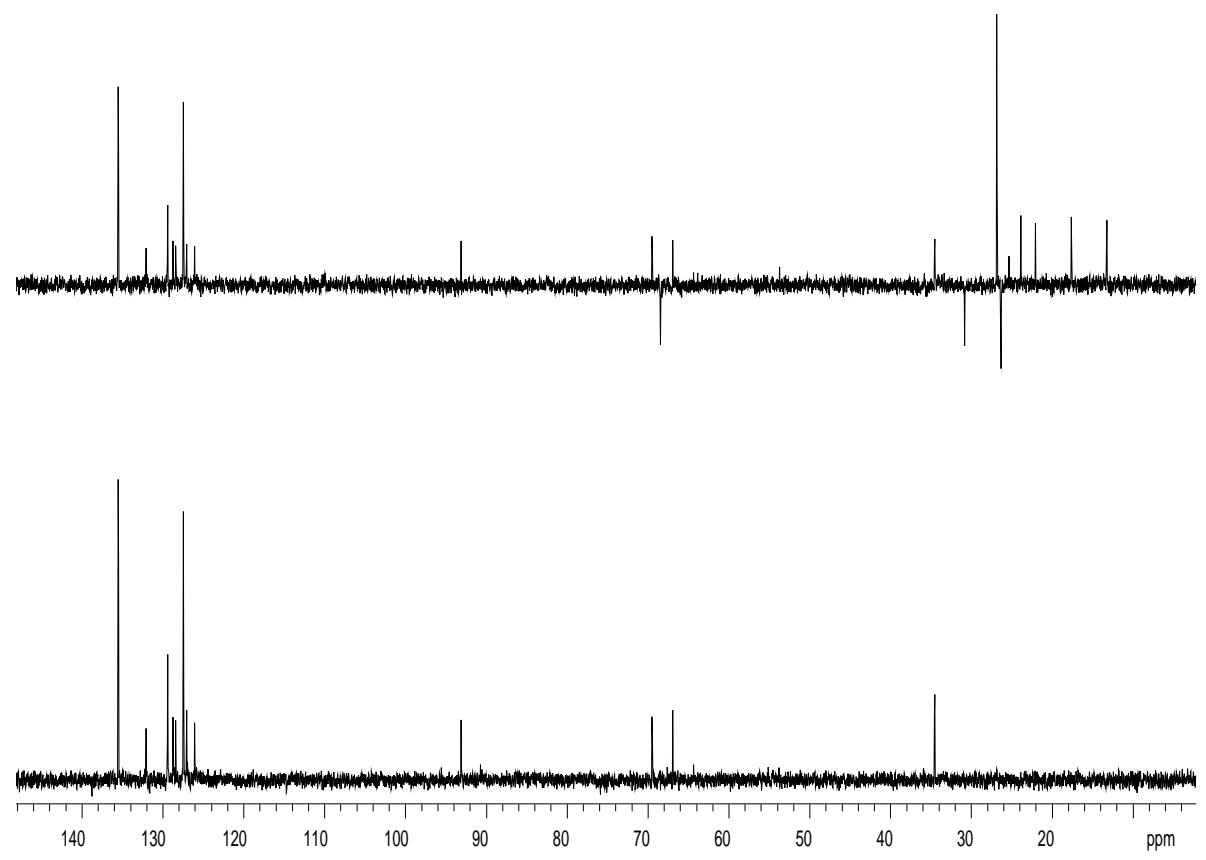

DEPT 90/135 (75 MHz, $\mathrm{CDCl}_{3}$ ) - Diene (22) 
SI-63

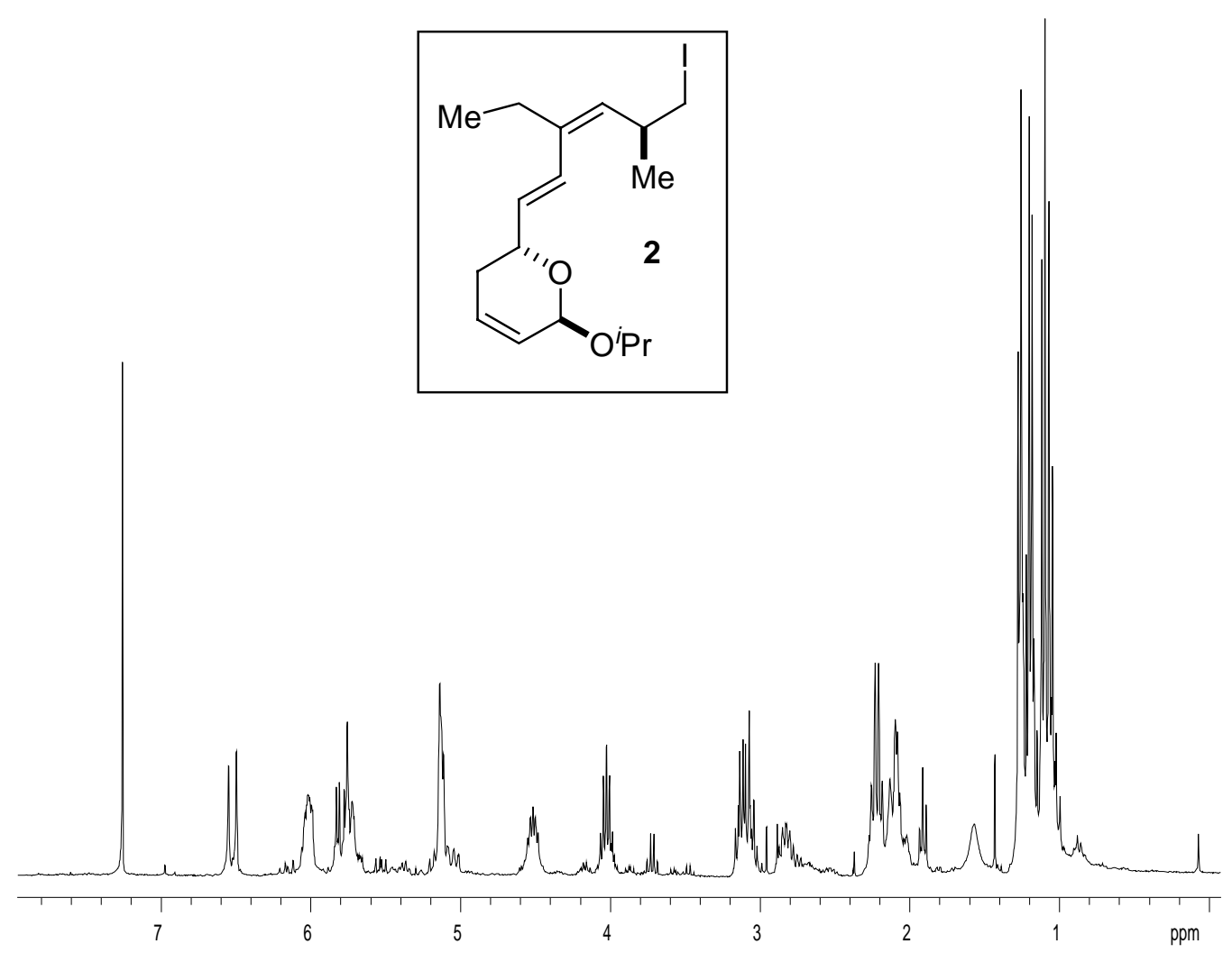

${ }^{1} \mathrm{H}$ NMR (300 MHz, $\mathrm{CDCl}_{3}$ ) - Alkyl iodide (2)

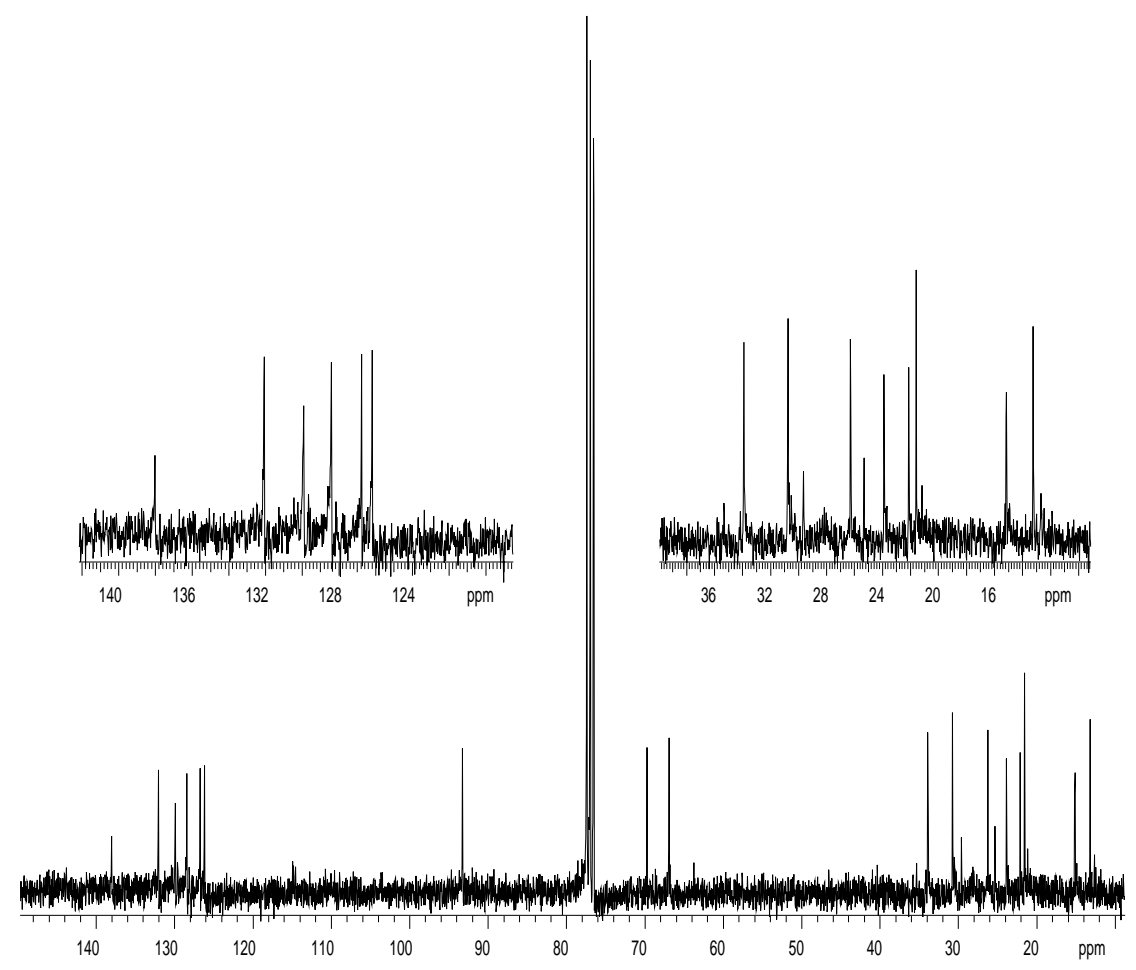

${ }^{13} \mathrm{C}$ NMR (75 MHz, $\mathrm{CDCl}_{3}$ ) - Alkyl iodide (2) 